\title{
Harnessing Interference for Analog Function Computation in Wireless Sensor Networks
}

\author{
Mario Goldenbaum, Student Member, IEEE, Holger Boche, Fellow, IEEE, and \\ Sławomir Stańczak, Senior Member, IEEE
}

\begin{abstract}
It is known that if the objective of a wireless sensor network is not to reconstruct individual sensor readings at a fusion center but rather to compute a linear function of them, then the interference property of the wireless channel can be beneficially harnessed by letting nodes transmit simultaneously. Recently, an analog computation scheme was proposed to show that it is possible to take the advantage of the interference property even if nonlinear functions are to be computed. The scheme involves some pre-processing on the sensor readings and post-processing on the superimposed signals observed by the fusion center. Correspondingly, this paper provides a thorough base for a theory of analog-computing functions over wireless channels by specifying what is the maximum achievable. This means it is determined for networks of arbitrary topology which functions are generally analog-computable over the channel and how many wireless resources are needed. It turns out that the considerations are closely related to the famous 13th Hilbert problem and that analog-computations can be universally performed in the sense that the pre-processing at sensor nodes is independent of the function to be computed. Universality reduces the complexity of transmitters and the signaling overhead, and it is shown that this property is preserved if nodes leave or join the network. Analog-computability is therefore of high practical relevance as it allows for an efficient computation of functions in sensor networks.
\end{abstract}

Index Terms-Computation over multiple-access channels, wireless sensor networks, pre- and post-processing, 13th Hilbert problem.

\section{INTRODUCTION}

$\mathbf{O}$ NGOING advances in microelectronics and wireless networking make sensor networks highly attractive for a wide range of applications from different fields [1]. In con-

Manuscript received April 30, 2012; revised October 10, 2012, March 05, 2013, and June 06, 2013; accepted June 14, 2013. Date of publication July 11, 2013; date of current version September 04, 2013. The associate editor coordinating the review of this manuscript and approving it for publication was Dr. Tongtong Li. This work was supported in part by the German Research Foundation (DFG) within the priority program 1397 "Communications in Interference Limited Networks (COIN)" under Grants STA 864/3-2 and BO 1734/24-1. This work appeared in part at the 2011 Int. Symp. Wireless Commun. Syst. (ISWCS), at the 2012 Annu. Conf. Inf. Sci. and Syst. (CISS), and at the 2012 IEEE Int. Conf. Acoustics, Speech, Signal Process. (ICASSP).

M. Goldenbaum is with the Fachgebiet für Informationstheorie und theoretische Informationstechnik, Technische Universität Berlin, 10587 Berlin, Germany, and with the Lehrstuhl für Theoretische Informationstechnik, Technische Universität München, 80333 Munich, Germany (e-mail: mario.goldenbaum@tu-berlin.de).

H. Boche is with the Lehrstuhl für Theoretische Informationstechnik, Technische Universität München, 80333 Munich, Germany (e-mail: boche@tum. de).

S. Stańczak is with the Fraunhofer Institute for Telecommunications, Heinrich Hertz Institute, 10587 Berlin, Germany, and with the Fachgebiet für Informationstheorie und theoretische Informationstechnik, Technische Universität Berlin, 10587 Berlin, Germany (e-mail: slawomir.stanczak@hhi.fraunhofer.de).

Digital Object Identifier 10.1109/TSP.2013.2272921 trast to conventional wireless networks, in which the objective is to provide high-capacity end-to-end connections for data transfer, the most exciting applications for wireless sensor networks are satisfied with low data rates but require that sensor nodes are powered by batteries or by environmentally scavenged energy. Thus, energy-efficient networking protocols for low-complexity, low-cost and low-power consumption wireless connectivity are crucial for ensuring a long network lifetime. In order to enhance the network efficiency not only in terms of energy consumption but also in terms of reliability and sensing quality, it is vital to tailor wireless sensor network solutions to specific application needs. Recently, this paradigm shift has been drawing more and more attention when designing next generation cellular networks [2].

A vast number of applications for wireless sensor networks require an efficient computation of functions of spatially distributed sensor readings (e.g., mean temperature, maximum pressure) [3]. Current approaches rely on a digital design where sensor nodes quantize their measurements and transmit them as bit streams to assigned fusion centers. In order to reconstruct the individual sensor readings, access to the channel is usually coordinated by standard protocols such as Time-Division Multiple-Access (TDMA) or Carrier-Sense Multiple-Access (CSMA). These protocols avoid strong interference by allowing different nodes to transmit concurrently only if the resulting interference level is low enough. Once the sensor readings are reconstructed from the received signals, the fusion centers evaluate the desired function value.

Such approaches treat communication and computation as distinct processes: the underlying function computation (i.e., the application) is not adequately taken into account in the design of the communication protocols. As a consequence, wireless resources are wasted since the fusion centers are not interested in individual measurements but only in functions of them. In [4], Gastpar and Vetterli merge the processes of communication and computation by developing a novel analog (i.e., non-digital) joint source-channel communication scheme. The proposed scheme exploits the interference property of a Gaussian Multiple-Access Channel (MAC) ${ }^{1}$ by letting nodes transmit simultaneously to compute a linear function of the measurements much more efficiently over the channel.

The problem of computing nonlinear functions via the wireless MAC is addressed in [5], [6]. The key idea is to apply a pre-processing function to each sensor reading prior to transmission and a post-processing function operating on the received signal, which is a superposition of the signals transmitted by the

\footnotetext{
${ }^{1}$ To avoid confusion, we point out that in this paper the acronym MAC is not an abbreviation for "medium access control", which is also widely used in communications engineering.
} 
individual sensor nodes. The functions are to be chosen such that the resulting overall channel directly provides the desired nonlinear function of the measurements at its output. Several corresponding function examples of high practical relevance can already be found in [5], [6]. However, a complete characterization of the associated function space was left as an open problem. To provide a thorough base for a theory of analog computation over wireless channels, we address the problem in this paper and determine what is the maximum achievable. This means that we specify which functions are generally analog-computable over the channel in networks of arbitrary topology and how efficiently this can be done in terms of wireless resource consumption. The presented results indicate that harnessing interference for analog function computations has the potential to provide huge performance gains in comparison to standard approaches.

\section{A. Related Work}

The problem to be solved in [4] is to estimate a parameter of interest from multiple sensor observations that are corrupted by Gaussian noise. The proposed communication scheme performs significantly better than standard approaches because the underlying Gaussian MAC itself computes the optimal linear estimation function (i.e., the arithmetic mean). This observation initiated many research activities in signal processing that extended the analog joint source-channel approach to more general estimation problems such as those in [7]-[10]. The authors of [11] propose an analog coding scheme that exploits the interference property of the wireless channel to efficiently estimate a linear vector-valued function of some local measurements in a network with fading channels. Besides, it was recognized that harnessing interference can also be promising for solving certain detection problems [12]-[17], whereas a first experimental validation that non-orthogonal transmissions can increase the efficiency in some wireless computation problems can be found in [18], [19]. Roughly speaking, all of these schemes harness the natural interference property of wireless channels to efficiently compute a special function of the measurements.

The efficient computation of functions can be viewed as a fundamental building block for other sophisticated in-network processing such as gossip algorithms. Gossip algorithms want to distributively achieve a rapid consensus between the nodes of a network with respect to a function of the current sensor readings (see [20]-[24] and references therein).

The general computation problem in an information theoretical manner is addressed in for example [25], [26] with an emphasis on adequate source coding. On the other hand, the information theoretical problem of reliably computing some functions of sources over a MAC is considered in the seminal paper [27], in [28], and in [29] for linear channels only.

\section{B. Contributions and Paper Organization}

In order to allow an in-depth and rigorous analysis and to provide insights into the limits of analog function computation in wireless networks, we assume an idealized wireless MAC model. Based on this, we determine which functions are generally analog-computable at a single fusion center by harnessing the interference property of the wireless channel and how efficiently this can be done in terms of wireless resource consumption. It turns out that the achievable efficiency strongly depends on some properties imposed on the pre- and post-processing functions. For example, from an implementation point of view, continuity can be highly desirable but the continuity property requires in general additional wireless resources for computations over the channel. This is a consequence of a result proved by Kolmogorov [30] that solves the famous 13th Hilbert problem stated in 1900 [31]. In contrast to efficiency, analog computations over the channel can always be universally performed regardless of whether the pre-processing functions are continuous or not. Throughout the paper, universality refers to transmit strategies that are independent of the function to be computed such there is no need to inform the sensor nodes once the function to be computed must be changed.

Based on these results for networks with a single fusion center, we consider a generalized sensor network model consisting of multiple fusion centers, each of which aims to independently compute some function of the sensor readings of an arbitrary subset of nodes (i.e., clusters). It turns out that all the previous properties carry over to the generalized case except if pre- and post-processing functions are supposed to be continuous, in which case some coordination may be necessary. If, however, the fusion centers perform a simple additional post-processing step, then it is shown that the coordination is not required. We also show that the universality property is preserved under changing topologies as a result of nodes dropping out of the network (due to for instance failures or battery depletion) or nodes joining the network.

Practical computation schemes will suffer from several impairments such as fading, receiver noise, transmit power constraints and the lack of synchronization. Therefore, to demonstrate that the gained insights under the idealized channel model do not loose its validity when taking practical aspects into account, we finally provide some numerical examples in which analog computations are carried out over non-ideal wireless MACs.

The paper is organized as follows. Section II presents the system model and the problem statement. In Section III the space of analog-computable functions is characterized. Subsequently, in Section IV the more general problem of computing multiple functions at distinct fusion centers is considered, whereas Section V is devoted to study the behavior of analog-computations under varying network topologies. Section VI provides some remarks on computations over non-ideal channels and presents some numerical examples. Finally, Section VII concludes the paper.

\section{Notational Remarks}

The $k$-times Cartesian product of a space $A$ is written as $A^{k}$. The natural and real numbers are denoted by $\mathbb{N}, \mathbb{R}=(-\infty, \infty)$, and $\mathbb{E}:=[0,1] \subset \mathbb{R}$ is the closed unit interval. For convenience, we sometimes write points $\left(\xi_{1}, \ldots, \xi_{k}\right)$ of any space $A^{k}$ as vectors $\boldsymbol{\xi}$. The zero vector is denoted by $\mathbf{0}$ and the $i$ th unit vector in the $k$-dimensional Euclidean space by $\boldsymbol{e}_{i}$, that is the zero vector with a 1 at the $i$ th position, $1 \leq i \leq k$. The identity map on any set $A$ is described by $\operatorname{id}_{A}: A \rightarrow A$. Let $A^{k}$ be a compact metric space, then $\mathcal{C}^{0}\left[A^{k}\right]$ denotes in conjunction with the infinity norm $\|\cdot\|_{\infty}$ the Banach space of real-valued continuous functions of $k \in \mathbb{N}$ variables, defined on $A^{k}$. Furthermore, $\mathcal{F}\left[\mathbb{B}^{\ell}\right]$ denotes the space of every function $g: \mathbb{B}^{\ell} \rightarrow \mathbb{R}$ defined on $\mathbb{B}^{\ell} \subseteq \mathbb{R}^{\ell}, \ell \in \mathbb{N}$, and $\mathrm{E}\{\cdot\}$ is the expectation operator. 


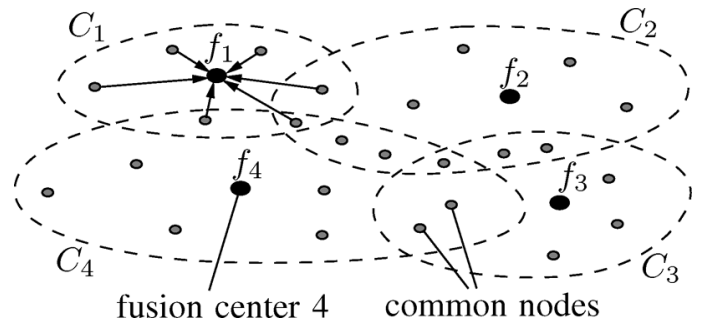

Fig. 1. A qualitative representation of a clustered wireless sensor network consisting of $N=25$ nodes and $K=4$ clusters for computing any functions $f_{1}, \ldots, f_{4}$ at fusion centers. Nodes belonging to any of the overlaps $C_{k} \cap C_{\ell}$, $k \neq \ell$, are called "common nodes".

\section{System Model AND Problem Statement}

Consider a wireless sensor network consisting of $N \in \mathbb{N}$ spatially distributed nodes that monitor the environment resulting in sensor readings $x_{n} \in \mathbb{E}, n=1, \ldots, N$. Assume that the network is organized into $K \in \mathbb{N}$ clusters, where the set of nodes belonging to cluster $k$ is denoted by $C_{k}, k=1, \ldots, K$, with $C_{k} \cap C_{\ell} \neq \varnothing$, for all $k, \ell$. We view each cluster, consisting of $\left|C_{k}\right|$ nodes, as a collection of distributed computation devices that aim to efficiently compute given real desired functions $f_{k}: \mathbb{E}^{\left|C_{k}\right|} \rightarrow \mathbb{R}, f_{k}\left(x_{k_{1}}, \ldots, x_{k_{\left|C_{k}\right|}}\right), k=1, \ldots, K$, of corresponding sensor readings at designated fusion centers (see Fig. 1).

To describe the intra-cluster communication between nodes and fusion centers, we use the standard affine model of a wireless MAC [32] such that the real-valued signal received by fusion center $k$ can be written as

$$
y_{k}(\ell)=\sum_{n \in C_{k}} h_{k n}(\ell) s_{n \ell}^{(k)}\left(x_{n}\right)+v_{k}(\ell), k=1, \ldots, K .
$$

Here and hereafter, $\ell=1, \ldots, L_{k} \in \mathbb{N}$ is a discrete time or a frequency sub-band, ${ }^{2} s_{n \ell}^{(k)}: \mathbb{E} \rightarrow \mathbb{R}$ denotes a transmit signal of node $n \in C_{k}$ depending on sensed value $x_{n}, h_{k n}(\ell) \in \mathbb{R}$ is a fading coefficient between node $n$ and fusion center $k$ and $v_{k}(\ell) \in \mathbb{R}$ is receiver noise, respectively. Ignoring in (1) the fading and the noise results then for $\ell=1, \ldots, L_{k}$ in $K$ ideal $M A C s$

$$
y_{k}(\ell)=\sum_{n \in C_{k}} s_{n \ell}^{(k)}\left(x_{n}\right)
$$

that interfere with each other due to common nodes (i.e., nodes which belong to more than one cluster such as illustrated in Fig. 1). The mappings (2) highlight superposition as the natural mathematical operation of a wireless MAC.

Remark 1: Note that the intuition behind (1) and (2) is that only the nodes belonging to cluster $C_{k}$ are able to reach the $k$ th fusion center, $k=1, \ldots, K$. This coincides with a scenario in which clusters are formed due to the connectivity radii of the $N$ spatially distributed nodes. In other words, there are $K$ independent fusion centers that aim at exploiting the public observations of all nodes to compute any function, but they are limited to node subsets due to reachability constraints.

\footnotetext{
${ }^{2}$ Or any other available wireless resource unit such as for example "beam direction" in a spatial-multiplexing system [33].
}

In contrast to standard medium-access protocols that are designed to avoid simultaneous transmissions in the same frequency band, the interference (2) can profitably be harnessed if fusion centers are interested in computing linear functions of the measurements [4], [11]. To enable the computation of nonlinear desired functions by means of wireless MACs as well, we consider appropriate pre- and post-processing functions defined as follows [5].

Definition 1 (Pre-Processing Functions): We define the univariate functions $\varphi_{n \ell}^{(k)}: \mathbb{E} \rightarrow \mathbb{R}$, operating on the sensor readings $x_{n}, n \in C_{k}$, at resource unit $\ell, \ell=1, \ldots, L_{k}, k=$ $1, \ldots, K$ (i.e., $\left.\varphi_{n \ell}^{(k)}\left(x_{n}\right)\right)$, to be the pre-processing functions.

Definition 2 (Post-Processing Functions): Let $y_{k}(\ell) \in \mathbb{R}$ be the output of the wireless MAC according to (1). Then, we define the univariate functions $\psi_{k \ell}: \mathbb{R} \rightarrow \mathbb{R}, \ell=1, \ldots, L_{k}$, $k=1, \ldots, K$, operating on $y_{k}(\ell)$ (i.e., $\psi_{k \ell}\left(y_{k}(\ell)\right)$ ), to be the post-processing functions.

The definitions of pre- and post-processing functions lead us to the formal definition of an $f$-MAC of order $L$, which will be the main ingredient of our investigations in this paper.

Definition 3 ( $f$-MAC of Order $L$ ): Let $N, L \in \mathbb{N}$ be any finite numbers of nodes and resource units and let $\left\{\varphi_{n \ell}\right\}_{1 \leq n \leq N, 1 \leq \ell \leq L},\left\{\psi_{\ell}\right\}_{1 \leq \ell \leq L}$ be sets of pre- and post-processing functions. Then, we define a series

$$
\begin{aligned}
\left\{\psi_{1}(y(1))=\psi_{1}\left(\sum_{n=1}^{N} \varphi_{n 1}\left(x_{n}\right)\right)\right. & \\
\ldots, \psi_{L}(y(L)) & \left.=\psi_{L}\left(\sum_{n=1}^{N} \varphi_{n L}\left(x_{n}\right)\right)\right\}
\end{aligned}
$$

of $L$ post-processed ideal MAC outputs $y(1), \ldots, y(L)$ as an $f$-MAC of order $L$.

Obviously, the clustered sensor network described above is with (2) and $s_{n \ell}^{(k)} \equiv \varphi_{n \ell}^{(k)}$, for all $n, \ell, k$, a network of $f$-MACs of orders $L_{k}, k=1, \ldots, K$. This allows at fusion centers the analog computation of all desired functions $f_{1}, \ldots, f_{K}$ for which pre- and post-processing functions exist, as well as numbers $L_{1}, \ldots, L_{K}$ and functions $\Psi_{k}: \Lambda_{k} \subset \mathbb{R}^{L_{k}} \rightarrow \mathbb{R}$, such that the desired functions can be represented in the form

$$
\begin{aligned}
& f_{k}\left(x_{k_{1}}, \ldots, x_{k_{\left|C_{k}\right|}}\right) \\
& \quad=\Psi_{k}\left(\psi_{k 1}\left(y_{k}(1)\right), \ldots, \psi_{k L_{k}}\left(y_{k}\left(L_{k}\right)\right)\right) .
\end{aligned}
$$

The purpose of functions $\Psi_{k}$ is to appropriately combine at fusion centers the corresponding sequences of post-processed MAC outputs.

With the above definitions in hand, we are now in a position to identify the following three fundamental problem domains:

(P1) What is the space of functions that are analog-computable over $f$-MACs (i.e., which functions have a representation (3))?

(P2) What is the highest possible computation efficiency expressed in terms of the number of resource units $L_{k}, k=$ $1, \ldots, K$, that are necessary to compute such functions?

(P3) What are the properties of analog computation networks with regard to node complexity and coordination effort? 
In answering these questions it will turn out that universality will play an important role, which we define as follows.

Definition 4 (Universality): We say that analog computation over an $f$-MAC is universal with respect to some function space $\mathcal{A}\left[\mathrm{A}^{M}\right]$, if the pre-processing functions are universal. That is if there exist fixed pre-processing functions that can be used to compute every desired function $f \in \mathcal{A}\left[\mathbb{A}^{M}\right]$ having a representation (3).

Remark 2: Note that universality is a highly desirable property for all-purpose computation networks since it mainly defines the communication structure within the network as well as to what extent coordination is required. In other words, if pre-processing functions did not depend on the functions to be computed at the fusion centers, no additional feedback ${ }^{3}$ would be necessary if desired functions change.

Remark 3: Although we mainly consider in this paper computations over $f$-MACs (i.e., sequences of ideal MAC outputs) to focus on the fundamentals of analog computation problems, extensions to realistic MACs (1) can follow along similar lines as in [5], [6], [28], [34]. See Section VI for more details.

\section{AnAlog COMPutation Over $f$-MACs}

To give a precise answer to question (P1), we have to analyze the function space consisting of all functions possessing representations (3). Therefore, we start with the simplest network consisting of a single cluster (i.e., $K=1$ and $\left|C_{1}\right|=N$ ) to obtain the first insights. The case of arbitrary clustered networks is considered in Section IV.

\section{A. Computations Over $f$-MACs of Order 1}

Let a single wireless resource unit be available for the computation of a function value (i.e., $L_{1}=L=1$ ). Then, the space consisting of functions (3) simplifies to

$$
\begin{aligned}
& \left\{f \in \mathcal{F}\left[\mathbb{E}^{N}\right] \mid \exists\left(\varphi_{1}, \ldots, \varphi_{N}, \psi\right) \in \mathcal{F}[\mathbb{E}] \times \cdots\right. \\
& \left.\cdots \times \mathcal{F}[\mathbb{E}] \times \mathcal{F}[\mathbb{R}]: f\left(x_{1}, \ldots, x_{N}\right)=\psi\left(\sum_{n=1}^{N} \varphi_{n}\left(x_{n}\right)\right)\right\} .
\end{aligned}
$$

Remark 4: Functions of (4) emphasize that pre- and post-processing functions transform an ideal MAC such that the resulting overall channel (i.e., an $f$-MAC of order 1) matches the structure of the desired function (see Fig. 2).

It is an interesting coincidence that the function space (4), essentially a conclusion from the natural interference property of the wireless channel, is in mathematics known as the space of nomographic functions [35], which we denote in the following by $\mathcal{N}\left[\mathbb{E}^{N}\right]$. The functions are called nomographic functions since they are the basis of nomographs. Nomographs are graphical representations which are useful for solving certain types of equations [36]. A popular example is the Smith Chart, often used in microwave engineering.

Example 1 (Nomographic Functions) (i) Arithmetic Mean: $f\left(x_{1}, \ldots, x_{N}\right)=\frac{1}{N} \sum_{n} x_{n}$, with $\varphi_{n}(x)=x$, for all $n=1, \ldots, N$, and $\psi(y)=y / N$. (ii) Euclidean Norm: $f\left(x_{1}, \ldots, x_{N}\right)=\sqrt{x_{1}^{2}+\cdots+x_{N}^{2}}$, with $\varphi_{n}(x)=x^{2}$, for all

\footnotetext{
${ }^{3}$ Additional to the feedback that is mandatory in practical wireless systems (e.g., for providing channel state information).
}

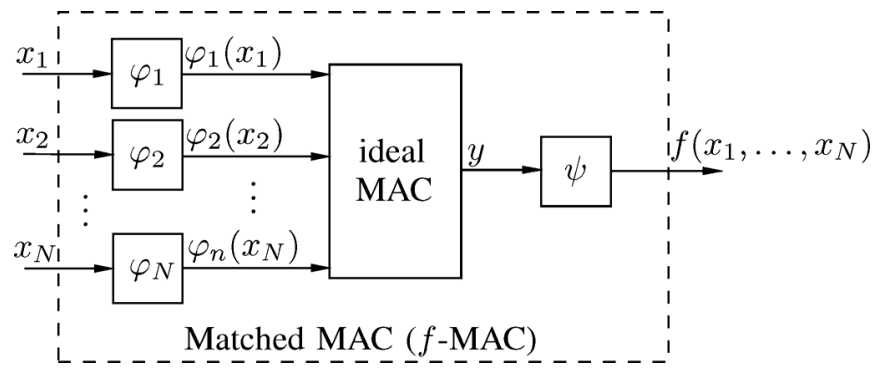

Fig. 2. $f$-MAC of order 1 with $x_{1}, \ldots, x_{N}$ as inputs and $f\left(x_{1}, \ldots, x_{N}\right)$ as output. That is an ideal MAC matched to the desired function $f$ by appropriate pre-processing functions $\varphi_{1}, \ldots, \varphi_{N}$ and a post-processing function $\psi$.

$n=1, \ldots, N$, and $\psi(y)=\sqrt{y}$. (iii) Number of Active Nodes: $f\left(x_{1}, \ldots, x_{N}\right)=N$, with $\varphi_{n}(x) \equiv 1$, for all $n=1, \ldots, N$, and $\psi(y)=y$.

The observation that (4) is exactly the space of nomographic functions leads us immediately to the following powerful theorem that entirely answers questions (P1) and (P2).

Theorem 1: Every desired function $f: \mathbb{E}^{N} \rightarrow \mathbb{R}$ is universally computable via an $f$-MAC of order 1 .

Proof: The proof results from [35] where it is shown that every $f \in \mathcal{F}\left[\mathbb{E}^{N}\right]$ has a nomographic representation

$$
f\left(x_{1}, \ldots, x_{N}\right)=\psi(\sum_{n=1}^{N} \underbrace{2^{n-1} \varphi\left(x_{n}\right)}_{=: \varphi_{n}\left(x_{n}\right)}),
$$

with a monotonically increasing $\varphi: \mathbb{E} \rightarrow \mathbb{E}$ that is independent of $f$ (i.e., universal). As a consequence, $\mathcal{N}\left[\mathbb{E}^{N}\right]=\mathcal{F}\left[\mathbb{E}^{N}\right]$.

Observation 1: The pre-processing at sensor nodes is independent of the desired function to be computed at the fusion center such that no additional feedback is necessary to inform the nodes about changes of the desired function. The fusion center decides by an appropriate choice of $\psi$ which $f$ shall be computed.

From the observation we conclude that the universality property offers the potential to significantly reduce in practice the amount of coordination as well as the hardware complexity of nodes. In Section IV, the property will play a key role in improving the efficiency of more general computation networks.

Remark 5: A theorem similar to Theorem 1 that is limited to continuous desired functions can be proven by using a result from [37].

Note that in Theorem 1 there are no restrictions on pre- and post-processing functions imposed. Since continuity can be advantageous for practical implementations, it is interesting to ask if Theorem 1 is also true if pre- and post-processing functions are required to be continuous. In this regard we denote in contrast to $\mathcal{N}\left[\mathbb{E}^{N}\right]$ the space of nomographic functions with the additional property $\left(\varphi_{1}, \ldots, \varphi_{N}, \psi\right) \in \mathcal{C}^{0}[\mathbb{E}] \times \cdots \times \mathcal{C}^{0}[\mathbb{E}] \times \mathcal{C}^{0}[\mathbb{R}]$ as $\mathcal{N}^{0}\left[\mathbb{E}^{N}\right]$. The following Lemma gives in conjunction with Observation 2 a first answer to this question by providing a necessary and sufficient condition for universality in the sense of Definition 4.

Lemma 1: Let $N \geq 2$ be arbitrary but fixed and let $g: \mathbb{E}^{N} \rightarrow$ $\Upsilon, g(\boldsymbol{x}):=g\left(x_{1}, \ldots, x_{N}\right)=\sum_{n=1}^{N} \varphi_{n}\left(x_{n}\right)$, with $\Upsilon \subseteq \mathbb{R}$ denoting the range of $g$. Then, $\left\{\varphi_{n}\right\}_{1 \leq n \leq N}$ are universal preprocessing functions for computing every $f \in \mathcal{F}\left[\mathbb{E}^{N}\right]$ if and only if the function $g$ is bijective. 


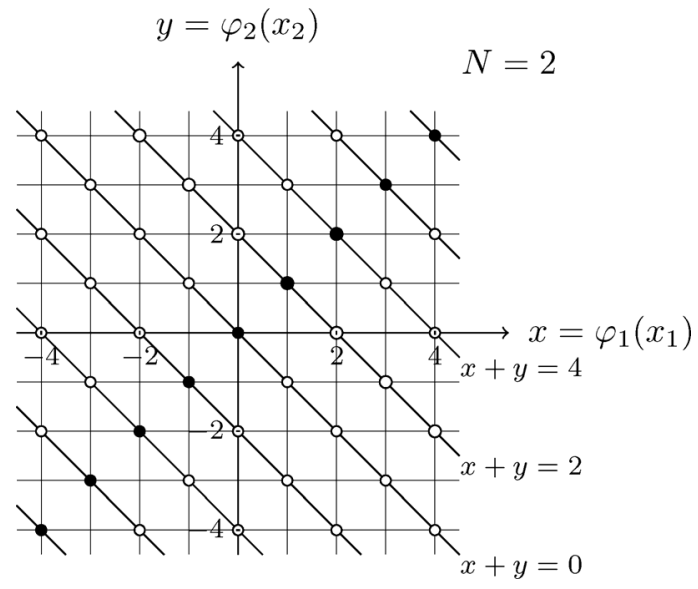

Fig. 3. A line grid of points $\varphi_{1}\left(x_{1}\right)+\varphi_{2}\left(x_{2}\right)=$ const. to illustrate the bijectivity requirement on function $g$. The pre-processing functions have to be chosen such that $\forall\left(x_{1}^{(1)}, x_{2}^{(1)}\right) \neq\left(x_{1}^{(2)}, x_{2}^{(2)}\right): \varphi_{1}\left(x_{1}^{(1)}\right)+\varphi_{2}\left(x_{2}^{(1)}\right) \neq$ $\varphi_{1}\left(x_{1}^{(2)}\right)+\varphi_{2}\left(x_{2}^{(2)}\right)$. That means in the depicted example that only the black points are allowed in the range of $g$ whereas the whites have to be avoided.

Proof: The proof is deferred to Appendix A.

Observation 2: For the function $g$ to be bijective, the preprocessing functions $\varphi_{1}, \ldots, \varphi_{N}$ have to be chosen in such a way that for all $\boldsymbol{x}^{(1)}, \boldsymbol{x}^{(2)} \in \mathbb{E}^{N}, \boldsymbol{x}^{(1)} \neq \boldsymbol{x}^{(2)}$, always

$$
\sum_{n=1}^{N} \varphi_{n}\left(x_{n}^{(1)}\right) \neq \sum_{n=1}^{N} \varphi_{n}\left(x_{n}^{(2)}\right)
$$

holds (see Fig. 3), which means that the ranges of the pre-processing functions have to be appropriate. To illustrate that this is possible, we consider the special case $N=2$ and construct a field $\mathbb{K} \subset \mathbb{R}$ which has the cardinality of the continuum without containing every real number. ${ }^{4}$ More precisely, we consider a real number $\alpha$ which is not in $\mathbb{K}$ (i.e., $\alpha \in \mathbb{R} \backslash \mathbb{K}$ ) and define $\mathbb{K}$ as the range of $\varphi_{1}$. Furthermore, we define the range of $\varphi_{2}$ to be the field $\frac{1}{\alpha} \mathbb{K}$. Then, for every $\left(x_{1}^{(1)}, x_{2}^{(1)}\right),\left(x_{1}^{(2)}, x_{2}^{(2)}\right) \in \mathbb{E}^{2}$, $\left(x_{1}^{(1)}, x_{2}^{(1)}\right) \stackrel{\alpha}{\neq}\left(x_{1}^{(2)}, x_{2}^{(2)}\right)$, it follows $\varphi_{1}\left(x_{1}^{(1)}\right)+\varphi_{2}\left(x_{2}^{(1)}\right) \neq$ $\varphi_{1}\left(x_{1}^{(2)}\right)+\varphi_{2}\left(x_{2}^{(2)}\right)$. Would this not be the case, then

$$
\begin{aligned}
\underbrace{\varphi_{1}\left(x_{1}^{(1)}\right)-\varphi_{1}\left(x_{1}^{(2)}\right)}_{\in \mathbb{K}} & =\varphi_{2}\left(x_{2}^{(2)}\right)-\varphi_{2}\left(x_{2}^{(1)}\right) \\
& =\frac{1}{\alpha} \underbrace{\left(y^{(2)}-y^{(1)}\right)}_{\in \mathbb{K}}
\end{aligned}
$$

would follow and thus $\alpha \in \mathbb{K}$, which would be a contradiction since $\alpha \in \mathbb{R} \backslash \mathbb{K}$.

Observation 2 unfortunately reveals that the necessary separation of all points in the range $\Upsilon$ of $g$ can never be achieved for every $f \in \mathcal{F}\left[\mathbb{E}^{N}\right]$ with continuous pre-processing functions since $\psi$ and $g$ are discontinuous in general. This observation is completed by the following theorem.

Theorem 2: The space of nomographic functions with continuous pre- and post-processing functions is nowhere dense in the space of continuous functions, that is $\mathcal{N}^{0}\left[\mathbb{E}^{N}\right]$ nowhere dense in $\mathcal{C}^{0}\left[\mathbb{E}^{N}\right]$.

\footnotetext{
${ }^{4}$ In [38], von Neumann constructs an example of such a field without using
} the axiom of choice.
Proof: The constructive proof for arbitrary $N$ is given by Buck in [39]. However, for the special case $N=2$ the theorem was previously proven by Arnol'd in [40].

Example 2 (Geometric Mean): Let $f: \mathbb{E}^{N} \rightarrow \mathbb{R}$ be the "geometric mean" $f\left(x_{1}, \ldots, x_{N}\right)=\left(\prod_{n} x_{n}\right)^{\frac{1}{N}}$ and let $\mathfrak{O}:=\left\{\left(x_{1}, \ldots, x_{N}\right) \in \mathbb{E}^{N} \mid \prod_{n=1}^{N} x_{n}=0\right\}$ be the closed subset of $\mathbb{E}^{N}$ on (and only on) which $f$ vanishes. Let $\mathfrak{E}:=\left\{\boldsymbol{e}_{1}, \ldots, \boldsymbol{e}_{N}\right\} \cup \mathbf{0}$ and suppose that there exist continuous pre- and post-processing functions such that $\left(\prod_{n} x_{n}\right)^{\frac{1}{N}}=\psi\left(\sum_{n} \varphi_{n}\left(x_{n}\right)\right)$ everywhere in $\mathbb{E}^{N}$. Any two of the $N+1$ points in $\mathfrak{E}$ can be connected by a polygonal line lying entirely in $\mathfrak{O}$ and by a polygonal line lying in the complement $\mathfrak{O}^{\mathrm{c}}:=\mathbb{E}^{N} \backslash \mathfrak{O}$ except at the end points. Now, assume that at the end points $\mathfrak{E}$, the continuous function $\sum_{n=1}^{N} \varphi_{n}\left(x_{n}\right)$ takes different values $\xi_{i}, i=1, \ldots, N+1$. Then, this leads to a contradiction because $\sum_{n=1}^{N} \varphi_{n}\left(x_{n}\right)$ would take the intermediate values $\frac{\xi_{i}+\xi_{j}}{2}, i, j=1, \ldots, N+1, i \neq j$, on $\mathfrak{O}$ and on $\mathfrak{O}^{\mathrm{c}}$ such that $\psi\left(\frac{\xi_{i}+\xi_{j}}{2}\right)=0$ and $\psi\left(\frac{\xi_{i}+\xi_{j}}{2}\right)>0$ simultaneously. ${ }^{5}$ We therefore conclude that $\sum_{n=1}^{N} \varphi_{n}\left(x_{n}\right)$ would take the same value at each $\left(x_{1}, \ldots, x_{N}\right) \in \mathfrak{E}$, from which $\varphi_{1}(0)+\cdots+\varphi_{N}(0)=\varphi_{1}(1)+\cdots+\varphi_{N}(1)$ follows as well as $\psi\left(\varphi_{1}(0)+\cdots+\varphi_{N}(0)\right)=\psi\left(\varphi_{1}(1)+\cdots+\varphi_{N}(1)\right)$. However, this is in contradiction to $0=f(0, \ldots, 0) \neq f(1, \ldots, 1)=1$ and we conclude that there do not exist continuous functions $\varphi_{1}, \ldots, \varphi_{N}, \psi$ which could ensure that the "geometric mean" is in $\mathcal{N}^{0}\left[\mathbb{E}^{N}\right]$.

\section{B. Approximations Over $f$-MACs of Order 1}

Although the continuity of pre- and post-processing functions reduces the amount of functions that are computable over an $f$-MAC of order 1 , some desired functions which are not nomographic according to $\mathcal{N}^{0}\left[\mathbb{E}^{N}\right]$ can still be computed if appropriate approximations by nomographic representations are allowed. This results in a multivariate approximation problem, specified more precisely in the following definition.

Definition 5 (Nomographic Approximations): Let $\varepsilon>0$ be arbitrary but fixed. Then, we define

$$
\begin{gathered}
\mathcal{N}_{\varepsilon}^{0}\left[\mathbb{E}^{N}\right]:=\left\{f \in \mathcal{F}\left[\mathbb{E}^{N}\right] \mid \exists\left(\varphi_{1}, \ldots, \varphi_{N}, \psi\right) \in \mathcal{C}^{0}[\mathbb{E}] \times \cdots\right. \\
\left.\cdots \times \mathcal{C}^{0}[\mathbb{E}] \times \mathcal{C}^{0}[\mathbb{R}]:\left\|f-\psi\left(\sum_{n} \varphi_{n}\left(x_{n}\right)\right)\right\|_{\infty} \leq \varepsilon\right\},
\end{gathered}
$$

as the space of approximable nomographic functions with respect to precision $\varepsilon$. If $f \in \mathcal{N}_{\varepsilon}^{0}\left[\mathbb{E}^{N}\right]$, we write $f\left(x_{1}, \ldots, x_{N}\right) \approx$ $\psi\left(\sum_{n} \varphi_{n}\left(x_{n}\right)\right)$.

An adequate characterization of any of the spaces $\mathcal{N}_{\varepsilon}^{0}\left[\mathbb{E}^{N}\right]$ is currently a serious problem. But if we go back to the specific Example 2, then there exists for every $\varepsilon>0$ a $p_{0}=p_{0}(\varepsilon)$ such that

$\forall p \geq p_{0} \forall \boldsymbol{x} \in \mathbb{E}^{N}:\left|\left(\prod_{n=1}^{N} x_{n}\right)^{\frac{1}{N}}-\mathrm{e}^{\frac{\sum_{n=1}^{N} \log _{\mathrm{e}}\left(x_{n}+\frac{1}{p}\right)}{N}}\right| \leq \varepsilon$.

${ }^{5} \mathrm{~A}$ continuous function on a continuum takes all values between any pair of given points. 
The desired function "geometric mean" is therefore the uniform limit of the sequence of nomographic functions

$$
\left\{\psi\left(\sum_{n} \varphi_{n_{p}}\left(x_{n}\right)\right)=\mathrm{e}^{\frac{1}{N} \sum_{n} \log _{e}\left(x_{n}+\frac{1}{p}\right)}\right\}_{p \in \mathbb{N}}
$$

with pre-processing functions $\varphi_{n_{p}}(x)=\log _{\mathrm{e}}\left(x+\frac{1}{p}\right), n=$ $1, \ldots, N$, and post-processing function $\psi(y)=\exp (y / N)$. In words, the "geometric mean", which is indeed an element of the function space $\mathcal{N}\left[\mathbb{E}^{N}\right]$ but unfortunately not of $\mathcal{N}^{0}\left[\mathbb{E}^{N}\right]$ (see Example 2), can be approximated with arbitrary precision by a nomographic function with continuous pre- and post-processing functions. Therefore, "geometric mean" is in any of the spaces $\mathcal{N}_{\varepsilon}^{0}\left[\mathbb{E}^{N}\right]$.

Remark 6: The example of "geometric mean" explicitly emphasizes that the wireless MAC is able to multiply.

Example 3 (Nomographic Approximations): Let $\varepsilon>0$ be arbitrary but fixed and let $p_{0}(\varepsilon)$ be chosen such that $f \in \mathcal{N}_{\varepsilon}^{0}\left[\mathbb{E}^{N}\right]$ for all $p \geq p_{0}(\varepsilon)$. (i) Cosine of the Product: $f\left(x_{1}, \ldots, x_{N}\right)=$ $\cos \left(\prod_{n} x_{n}\right) \approx \psi\left(\sum_{n} \varphi_{n}\left(x_{n}\right)\right)$, with $\varphi_{n}(x)=\log _{\mathrm{e}}\left(x+\frac{1}{p_{0}(\varepsilon)}\right)$, for all $n=1, \ldots, N$, and $\psi(y)=(\cos \circ \exp )(y)=\cos \left(\mathrm{e}^{y}\right)$. (ii) Maximum Value: $f\left(x_{1}, \ldots, x_{N}\right)=\max _{1<n<N}\left\{x_{n}\right\} \approx$ $\psi\left(\sum_{n} \varphi_{n}\left(x_{n}\right)\right)$, with $\varphi_{n}(x)=x^{p_{0}(\varepsilon)}$, for all $n=1, \ldots, N$, and $\psi(y)=y^{\frac{1}{p_{0}(\varepsilon)}}$. (iii) Minimum Value: $f\left(x_{1}, \ldots, x_{N}\right)=$ $\min _{1 \leq n \leq N}\left\{x_{n}\right\} \approx \psi\left(\sum_{n} \varphi_{n}\left(x_{n}\right)\right)$, with $\varphi_{n}(x)=\frac{1}{x^{p_{0}(\varepsilon)}}$, for all $n=1, \ldots, N$, and $\psi(y)=y^{-\frac{1}{p_{0}(\varepsilon)}}$.

Even if the desired functions in Example 3 are not universal nomographic approximations, in particular Examples 3 (ii) and (iii) can be relevant for alarm-driven sensor network applications. To make this more precise suppose a wireless sensor network is used for fire detection in a factory by periodically computing the maximal temperature (or carbon monoxide concentration) at a fusion center and comparing the result with a predefined threshold. Due to safety reasons this has to be done with a minimum transmission delay, which can be achieved within a single channel use by letting all nodes transmit their pre-processed measurements simultaneously to approximate the maximum over the channel in the sense of Example 3 (ii).

Remark 7: Note that the nomographic functions and approximations in Examples 1 and 3 do not consist of universal pre-processing functions, from which it is obvious that such representations are not necessarily unique. Since the results of this section that refer to universality are existence statements, finding universal pre-processing functions for computations over an $f$-MAC of order 1 poses a challenge for future work.

\section{Computations Over f-MACs of Order Greater Than 1}

In the previous Sections III-A and III-B we have seen that the continuity of pre- and post-processing functions crucially impacts the space of computable functions. In particular, an $f$-MAC of order 1 was not sufficient to compute every $f \in$ $\mathcal{C}^{0}\left[\mathbb{E}^{N}\right]$ as long as pre- and post-processing functions are required to be continuous. To get a more complete understanding of this behavior, we extend our single cluster considerations in this section to an $f$-MAC of order $L>1$. This allows for further harnessing the interference property of wireless channels by having more degrees of freedom such that we are now interested in characterizing the space of desired functions that are representable as (cf. (3))

$$
f\left(x_{1}, \ldots, x_{N}\right)=\Psi\left(\psi_{1}(y(1)), \ldots, \psi_{L}(y(L))\right),
$$

with continuous pre- and post-processing functions.

It is interesting to realize that this question is closely related to the 13th of the famous 23 problems stated by Hilbert in 1900 [31], [41]. The original problem involves the study of solutions of algebraic equations and Hilbert conjectured that a solution of the general equation of degree seven cannot be represented as a superposition of continuous functions of two variables.

In our context, Hilbert's conjecture implies that based on an $f$-MAC of finite order, the computation of every continuous desired function is not possible. Fortunately, the conjecture was disproved by Kolmogorov in his landmark paper [30]. We use a remarkable refinement of Kolmogorov's result to state the following theorem, which gives in contrast to Theorem $1 \mathrm{a}$ complete answer to questions (P1) and (P2) under the continuity requirement.

Theorem 3: With continuous pre-processing functions, every continuous desired function of $N$ variables is universally computable over an $f$-MAC of order $2 N+1$.

Proof: The proof follows from [42] where it is constructively shown that every $f \in \mathcal{C}^{0}\left[\mathbb{E}^{N}\right]$ is representable as

$$
f\left(x_{1}, \ldots, x_{N}\right)=\sum_{\ell=1}^{2 N+1} \zeta_{\ell}\left(x_{1}, \ldots, x_{N}\right)
$$

with $\zeta_{\ell} \in \mathcal{N}^{0}\left[\mathbb{E}^{N}\right], \ell=1, \ldots, 2 N+1$, defined as

$$
\zeta_{\ell}\left(x_{1}, \ldots, x_{N}\right):=\psi_{\ell}\left(\sum_{n=1}^{N} \alpha_{n} \varphi\left(x_{n}+(\ell-1) \beta\right)\right) .
$$

Here, $\varphi$ is a well defined, continuous and monotone increasing function and $\left\{\alpha_{n}\right\}_{n=1}^{N}, \beta$ are appropriate nonnegative real constants. Only the post-processing functions $\psi_{\ell} \in \mathcal{C}^{0}[\mathbb{R}], \ell=$ $1, \ldots, 2 N+1$, depend on $f$ but the $N(2 N+1)$ continuous pre-processing functions $\varphi_{n \ell}:=\alpha_{n} \varphi\left(x_{n}+(\ell-1) \beta\right)$ do not.

Representation (9) reveals that $\Psi: \Lambda \subset \mathbb{R}^{2 N+1} \rightarrow \mathbb{R}$ in (8) can be chosen to be for every $f \in \mathcal{C}^{0}\left[\mathbb{E}^{N}\right]$ simply the sum over the $f$-MAC of order $2 N+1$ output, that is $\Psi\left(\tilde{y}_{1}, \ldots, \tilde{y}_{2 N+1}\right)=$ $\sum_{\ell=1}^{2 N+1} \tilde{y}_{\ell}$, with $\tilde{y}_{\ell}:=\zeta_{\ell}\left(x_{1}, \ldots, x_{N}\right)=\psi_{\ell}(y(\ell))$.

Geometrically, Theorem 3 states that using $2 N+1$ distinct wireless resources (see Definition 3 ) results in a continuous and bijective correspondence $\left(x_{1}, \ldots, x_{N}\right) \mapsto(y(1), \ldots, y(2 N+$ 1)),

$$
\left(\begin{array}{c}
y(1) \\
y(2) \\
\vdots \\
y(2 N+1)
\end{array}\right)=\left(\begin{array}{c}
\sum_{n=1}^{N} \alpha_{n} \varphi\left(x_{n}\right) \\
\sum_{n=1}^{N} \alpha_{n} \varphi\left(x_{n}+\beta\right) \\
\vdots \\
\sum_{n=1}^{N} \alpha_{n} \varphi\left(x_{n}+2 N \beta\right)
\end{array}\right) \in \Gamma
$$

between sensor readings and ideal MAC output-signals $y(\ell)$, $\ell=1, \ldots, 2 N+1$, with $\Gamma$ a compact subset of the Euclidean space $\mathbb{R}^{2 N+1}$. In other words, (11) describes a homeomorphism between $\mathbb{E}^{N}$ and $\Gamma$ such that $\mathbb{E}^{N}$ is continuously embedded into $\mathbb{R}^{2 N+1}$. Hence, there exists a bijective correspondence between 
all continuous functions $f\left(x_{1}, \ldots, x_{N}\right)$ on $\mathbb{E}^{N}$ and all continuous functions $F(y(1), \ldots, y(2 N+1))$ on $\Gamma$.

Remark 8: Due to Kolmogorov's refutation of Hilbert's 13th problem, the $f$-MAC of order $2 N+1$ in Theorem 3 would initially be sufficient. In algebraic topology it was previously known from the Menger-Nöbeling theorem [43] that any compact space of dimension $N$ is homeomorphic to a subset of $\mathbb{R}^{2 N+1}$. However, the fact that particular compact spaces can be embedded into spaces of dimension lower than $2 N+1$ (e.g., $\mathbb{E}^{N}$ can be embedded into $\mathbb{R}^{N}$ by the identity map) suggests that maybe the $f$-MAC order in Theorem 3 can be decreased to save wireless resources. Unfortunately, it was proven in [44] that this is not possible (i.e., $L \geq 2 N+1$ is necessary to compute every $f \in \mathcal{C}^{0}\left[\mathbb{E}^{N}\right]$ with continuous pre- and post-processing functions). From [45], we conclude that if we further restrict pre-processing functions to be continuously differentiable, then Theorem 3 no longer holds (i.e., $2 N+1$ resource units are not sufficient for every continuous function). Roughly speaking, restrictions cost wireless resources or reduce the space of computable functions.

Although for $L \geq N$ there exist alternative approaches to appropriately compute desired functions over sensor networks (e.g., in an ideal TDMA protocol the entire analog sensor readings are conveyed interference free to the fusion center), the computation over $f$-MACs of order $L=2 N+1$ can lead to huge performance gains. Especially when the network consists of $K>1$ clusters, which is shown in the next section. But first, we summarize the main results of Section III as follows:

- If no restrictions on pre- and post-processing functions are imposed, the space of functions analog-computable over an $f$-MAC of order 1 is $\mathcal{F}\left[\mathbb{E}^{N}\right]$.

- If pre- and post-processing functions are required to be continuous, the space of functions analog-computable over an $f$-MAC of order 1 is nowhere dense in $\mathcal{C}^{0}\left[\mathbb{E}^{N}\right]$.

- If pre- and post-processing functions are required to be continuous, the space of functions analog-computable over an $f$-MAC of order $2 N+1$ is $\mathcal{C}^{0}\left[\mathbb{E}^{N}\right]$.

\section{Analog Computation Over Networked $f$-MACs}

As the above summary shows, we were able to completely answer questions (P1) and (P2). Now, we use the obtained insights to treat the remaining problem (P3) and consider therefore the general case in which the network consists of $K>1$ clusters as described in Section II (see Fig. 1 for a qualitative example). The aim of the network is to efficiently compute the $K$ desired functions (3) at the designated fusion centers, which is equivalent to efficiently compute the vector-valued function $f: \mathbb{E}^{N} \rightarrow \mathbb{R}^{K}$

$$
\boldsymbol{f}\left(x_{1}, \ldots, x_{N}\right):=\left(\begin{array}{c}
f_{1}\left(x_{1_{1}}, \ldots, x_{1_{\left|C_{1}\right|}}\right) \\
\vdots \\
f_{K}\left(x_{K_{1}}, \ldots, x_{K_{\left|C_{K}\right|}}\right)
\end{array}\right),
$$

over a network of $f$-MACs of orders $L_{1}, \ldots, L_{K}$. In doing so, we start in Section IV.A with the case where only continuous pre- and post-processing functions are allowed whereas in Section IV.B no restrictions on pre- and post-processing functions are imposed.

\section{A. Continuous Pre- and Post-Processing Functions}

If continuous pre- and post-processing functions are desired to facilitate the implementation in practical systems, we know from Theorem 3 that to universally compute every continuous function of $N \in \mathbb{N}$ sensor readings by means of the wireless channel, at least $L=2 N+1$ resource units are required.

The reason is that the $N$-dimensional space of sensor readings $\mathbb{E}^{N}$ has to be homeomorphically mapped onto a compact set $\Gamma$ of topological dimension $2 N+1$ (cf. (11)). Since this can be achieved by using an $f$-MAC of order $2 N+1$, we interpret it as collecting the required topological dimensions via distinct wireless resource units. But if the fusion center can only receive signals from a subset of the $N$ nodes (i.e., some summands on the right hand side of (11) are missing), then the image (11) is not necessarily in $\Gamma$, which in turn implies that not every $F \in \mathcal{C}^{0}[\Gamma]$ is computable. This is exactly what happens in a clustered network in which the nodes cannot reach all fusion centers (see Fig. 1) such that each component of $f$ depends only on a subset of the nodes.

Fortunately, due to the structural properties figured out in Section III.C this fact can be easily and independently solved at each fusion center. Let us therefore summarize the ideal MAC output-signals at the fusion centers to the vectors

$$
\boldsymbol{y}_{k}=\left(\begin{array}{c}
y_{k}(1) \\
\vdots \\
y_{k}(2 N+1)
\end{array}\right)=\left(\begin{array}{c}
\sum_{n \in C_{k}} \alpha_{n} \varphi\left(x_{n}\right) \\
\vdots \\
\sum_{n \in C_{k}} \alpha_{n} \varphi\left(x_{n}+2 N \beta\right)
\end{array}\right),
$$

$k=1, \ldots, K$, which are in general not points in $\Gamma$. However, if we consider the shifted versions

$$
z_{k}:=\boldsymbol{y}_{k}+\gamma_{k}
$$

with

$$
\boldsymbol{\gamma}_{k}=\left(\begin{array}{c}
\gamma_{k}(1) \\
\vdots \\
\gamma_{k}(2 N+1)
\end{array}\right):=\left(\begin{array}{c}
\sum_{n \notin C_{k}} \alpha_{n} \varphi(0) \\
\sum_{n \notin C_{k}} \alpha_{n} \varphi(\beta) \\
\vdots \\
\sum_{n \notin C_{k}} \alpha_{n} \varphi(2 N \beta)
\end{array}\right)
$$

then unlike $\boldsymbol{y}_{k}, z_{k}=\left(z_{k}(1), \ldots, z_{k}(2 N+1)\right)$ is a member of $\Gamma$, for all $k=1, \ldots, K$. It is important to emphasize that $\gamma_{k} \in \mathbb{R}^{2 N+1}$ is a constant and therefore independent of the sensor readings.

Now, the function to be computed at any fusion center does not depend on sensor readings of the remaining clusters. But this is not a limitation at all, since we are able to conclude from (14) and (15) a simple post-processing at fusion centers. The additional step enables the efficient computation of every vector-valued function consisting of component-functions $f_{k} \in$ $\mathcal{C}^{0}\left[\mathbb{E}^{\left|C_{k}\right|}\right]$ over a network of $f$-MACs of order $2 N+1$, provided that each fusion center a priori knows the number $N$ of nodes in the network. So, after receiving on the $\ell$ th resource unit the MAC output-signal $y_{k}(\ell)$ and after adding the correction term 


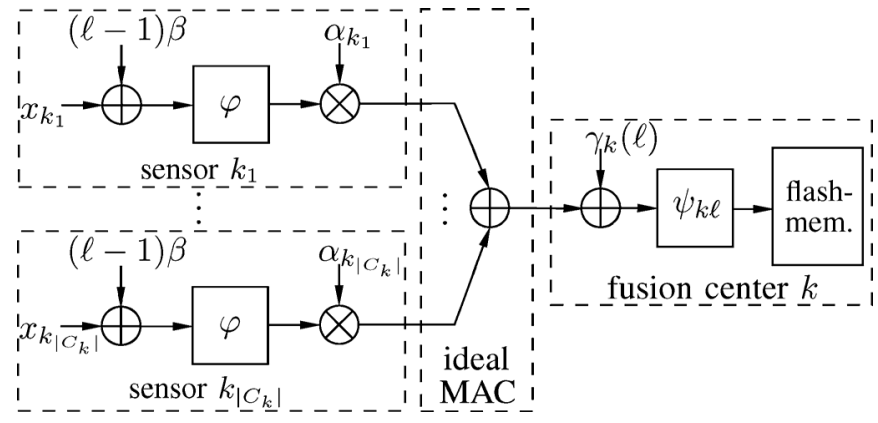

Fig. 4. Block diagram for computations in cluster $k, k=1, \ldots, K$, at wireless resource unit $\ell, \ell=1, \ldots, 2 N+1$, consisting of $\left|C_{k}\right|$ transmitting sensor nodes and a fusion center. At the end, the fusion center has to sum up all $2 N+1$ receive-signals from the flash memory which results immediately in the desired function value $f_{k}\left(x_{k_{1}}, \ldots, x_{k_{\left|C_{k}\right|}}\right)$.

$\gamma_{k}(\ell)$, fusion center $k, k=1, \ldots, K$, applies the corresponding post-processing function to $z_{k \ell}$

$$
\psi_{k \ell}\left(z_{k \ell}\right)=\psi_{k \ell}\left(\sum_{n \in C_{k}} \alpha_{n} \varphi\left(x_{n}+(\ell-1) \beta\right)+\gamma_{k}(\ell)\right),
$$

and stores this intermediate result in a memory. Finally, if all $2 N+1$ MAC output-signals are received and post-processed, the fusion centers compute the desired component-functions by summing up the respective memory content to obtain

$$
\boldsymbol{f}\left(x_{1}, \ldots, x_{N}\right)=\left(\begin{array}{c}
\sum_{\ell=1}^{2 N+1} \psi_{1 \ell}\left(z_{1}(\ell)\right) \\
\vdots \\
\sum_{\ell=1}^{2 N+1} \psi_{K \ell}\left(z_{K}(\ell)\right)
\end{array}\right) .
$$

A corresponding block diagram for a particular resource unit $\ell, \ell=1, \ldots, 2 N+1$, and a particular fusion center $k, k=$ $1, \ldots, K$, is depicted in Fig. 4.

Remark 9: Since the pre-processing functions are independent of the components of $\boldsymbol{f}$, the fusion centers determine by appropriately choosing the $K(2 N+1)$ post-processing functions $\left\{\psi_{k \ell}\right\}$, which continuous functions $f_{1}, \ldots, f_{K}$ are to be universally computed. Moreover, the constructive proof of Theorem 3 in [42] provides an algorithm that can be used to determine the universal pre-processing function $\varphi$ as well as the constants $\left\{\alpha_{n}\right\}, \beta$.

Remark 10: Note that the constants $\alpha_{n}$ need not to be different for all $N$ nodes in the network such that they can be reused in different clusters without any kind of arrangement between them.

In summary, we can state that with the above described postprocessing, all properties of Section III.C carry over to arbitrary clustered sensor networks. The resulting conclusions to answer question (P3) are discussed in Section IV-D.

\section{B. Arbitrary Pre- and Post-Processing Functions}

If no restrictions on pre- and post-processing functions are imposed, the situation is much less complicated than in the last subsection, since from Theorem 1 we conclude that already a single simultaneous transmission of the nodes in each cluster is sufficient to universally compute every

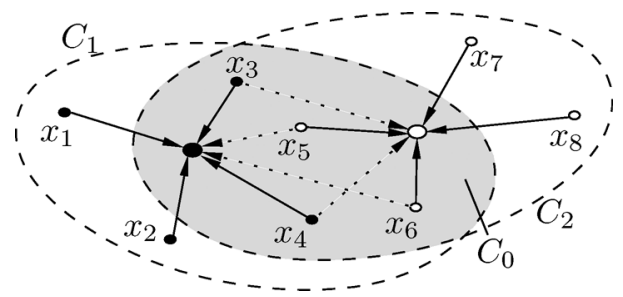

Fig. 5. Two interfering (i.e., overlapping) sensor networks. Sets $C_{1}$ and $C_{2}$ summarize the nodes belonging to network one and two, whereas the dashed regions are the connectivity radii of the corresponding fusion centers, such that signals transmitted by nodes in the shaded overlap $C_{0}$ are received at both fusion centers.

$f \in \mathcal{F}\left[\mathbb{E}^{\left|C_{1}\right|}\right] \times \cdots \times \mathcal{F}\left[\mathbb{E}^{\left|C_{K}\right|}\right]$. Consider therefore the $K$ networked ideal MAC output-signals

$$
y_{k}=g_{k}\left(x_{k_{1}}, \ldots, x_{k_{\left|C_{k}\right|}}\right)=\sum_{n \in C_{k}} \varphi_{n}\left(x_{n}\right)
$$

received by the fusion centers, which are mappings $g_{k}: \mathbb{E}^{\left|C_{k}\right|} \rightarrow \Upsilon_{k} \subset \mathbb{R}, k=1, \ldots, K$. Then, the post-processing at fusion centers consists merely in the application of appropriate post-processing functions such that every $f \in \mathcal{F}\left[\mathbb{E}^{\left|C_{1}\right|}\right] \times \cdots \times \mathcal{F}\left[\mathbb{E}^{\left|C_{K}\right|}\right]$ can be represented as

$$
\boldsymbol{f}\left(x_{1}, \ldots, x_{N}\right)=\left(\begin{array}{c}
\psi_{1}\left(y_{1}\right) \\
\vdots \\
\psi_{K}\left(y_{K}\right)
\end{array}\right) .
$$

But what is the difference to the previous case where continuous pre- and post-processing functions are desired?

We conclude from Lemma 1 that a necessary and sufficient condition to universally compute every vector-valued function (19) is that the functions $g_{k}, k=1, \ldots, K$, defined in (18), are bijective. Since this can never be achieved for every $f$ with continuous pre- and post-processing functions, it was necessary in Section III.C to appropriately embed $\mathbb{E}^{N}$ into a higher dimensional space, resulting in a bijection between function spaces $\mathcal{C}^{0}\left[\mathbb{E}^{N}\right]$ and $\mathcal{C}^{0}[\Gamma]$ instead. If pre- and post-processing functions are allowed to be discontinuous, however, such an embedding is superfluous.

\section{A Note About Additional Interference}

In Remark 1 we pointed out that the transmission model (see (1) and (2)) has to be understood in the sense that clusters are formed due to the connectivity radii of sensor nodes such that the overlap between clusters is determined by the spatial position of fusion centers. Thus, the fusion centers compute functions of subsets of freely accessible measurements. The results of this section, however, also remain valid in a scenario in which distinct computation sensor networks interfere with each other. To illustrate this, consider without loss of generality the example depicted in Fig. 5 consisting of two interfering sensor systems deployed to compute functions $f_{1}$ and $f_{2}$. More precisely, let $C_{1}$ and $C_{2}$ be the finite sets of nodes belonging to systems one and two, respectively, and let the observations of the corresponding nodes be summarized in the independent vectors $\boldsymbol{x}_{1}=$ $\left(x_{1}, \ldots, x_{\left|C_{1}\right|}\right) \in \mathbb{E}^{\left|C_{1}\right|}$ and $\boldsymbol{x}_{2}=\left(x_{\left|C_{1}\right|+1}, \ldots, x_{\left|C_{1}\right|+\left|C_{2}\right|}\right) \in$ $\mathbb{E}^{\left|C_{2}\right|}$. The difference to our previous studies (cf. (18)) is that the 
overlap region denoted as $C_{0}$ contains nodes from both systems, such that uncoordinated transmissions may result in signals

$$
y_{k}=\sum_{n \in C_{k}} \varphi_{n}\left(x_{n}\right)+\underbrace{\sum_{\substack{n \in C_{\ell} \cap C_{0} \\ \ell \neq k}} \varphi_{n}\left(x_{n}\right)}_{\text {interference }}, \quad k=1,2,
$$

received at the fusion centers.

First, it seems that the mutual interference prevents the adequate computation of arbitrary functions $f_{1}\left(\boldsymbol{x}_{1}\right)=$ $\psi_{1}\left(\sum_{n \in C_{1}} \varphi_{n}\left(x_{n}\right)\right), f_{2}\left(\boldsymbol{x}_{2}\right)=\psi_{2}\left(\sum_{n \in C_{2}} \varphi_{n}\left(x_{n}\right)\right)$. However, if fusion center one has knowledge about the interfering nodes $n \in C_{2} \cap C_{0}$ and fusion center two about the nodes $n \in C_{1} \cap C_{0}$, respectively, the problem is equivalent to the problem of computing functions of fewer variables as available. This knowledge provided, there always exist post-processing functions $\tilde{\psi}_{1}, \tilde{\psi}_{2} \in \mathcal{F}[\mathbb{R}]$ such that $\tilde{\psi}_{1}\left(y_{1}\right)=f_{1}\left(x_{1}, \ldots, x_{\left|C_{1}\right|}\right)$, for all $\boldsymbol{x}_{1} \in \mathbb{E}^{\left|C_{1}\right|}$ and all $\left\{x_{n} \in \mathbb{E}\right\}_{n \in C_{2} \cap C_{0}}$ as well as $\tilde{\psi}_{2}\left(y_{2}\right)=f_{2}\left(x_{\left|C_{1}\right|+1}, \ldots, x_{\left|C_{1}+\right| C_{2} \mid}\right)$, for all $\boldsymbol{x}_{2} \in \mathbb{E}^{\left|C_{2}\right|}$ and all $\left\{x_{n} \in \mathbb{E}\right\}_{n \in C_{1} \cap C_{0}}$. From Lemma 1 we already know that to achieve this, the pre-processing functions in both systems have to be chosen such that all $\boldsymbol{x}_{k}^{(1)}, \boldsymbol{x}_{k}^{(2)} \in \mathbb{E}^{\left|C_{k}\right|}$, with $\boldsymbol{x}_{k}^{(1)} \neq \boldsymbol{x}_{k}^{(2)}$, lead always to separated receive signals $y_{k}^{(1)} \neq y_{k}^{(2)}, k=1,2$.

Remark 11: If it is not possible to provide knowledge about nodes of interfering systems to the respective fusion centers, then the unwanted part of the interference has to be treated as an additional noise process. Considering this in detail will be part of future work.

\section{Performance Comparison}

To answer question (P3), we highlight in this section the advantages of the computation approach depicted in Fig. 4 in a network of $K$ clusters over standard TDMA protocols.

If no restrictions on pre- and post-processing functions are imposed, we conclude from Theorem 1 as well as from Section IV-B that in fact every function on each fusion center can be universally computed without significant coordination. This can be achieved by harnessing the natural interference property of wireless channels (i.e., without interference avoidance). The required number of resource units is of the order $\mathcal{O}(1)$ and therefore independent of the number of nodes and clusters. Alternatively, when a standard TDMA protocol is employed to compute functions at fusion centers in a clustered wireless sensor network, besides the orthogonalized medium-access of the nodes in each cluster, clusters themselves have to be appropriately separated in time, which requires a significant amount of coordination (see Fig. 6 for an illustration). A standard TDMA protocol would therefore induce $K M \geq K \max _{k}\left\{\left|C_{k}\right|\right\}$ separated transmissions to convey the entire raw sensor readings interference-free to the $K$ fusion centers, which subsequently compute the desired functions $f_{1}, \ldots, f_{K}$. Thus, it requires $\mathcal{O}(K M)$ wireless resource units. In contrast, we conclude from Section IV-A that $\mathcal{O}(2 N+1)$ wireless resource units are sufficient for computing every $f \in \mathcal{C}^{0}\left[\mathbb{E}^{\left|C_{1}\right|}\right] \times \cdots \times \mathcal{C}^{0}\left[\mathbb{E}^{\left|C_{K}\right|}\right]$ if pre- and post-processing functions are required to be continuous. Obviously, the number $2 N+1$ does not scales directly with the number of clusters such that huge performance gains are possible for $K>1$.

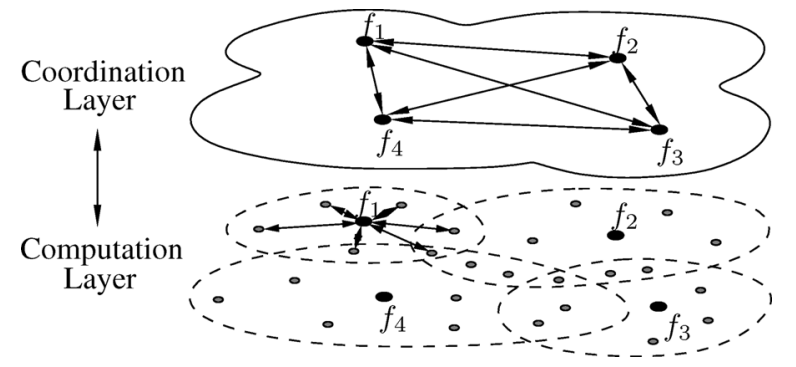

Fig. 6. The clustered sensor network example from Fig. 1 with an additional coordination layer that coordinates the medium-access of clusters such as in a standard TDMA approach. This requires bidirectional wireless communication links between the coordination layer and the computation layer as well as between the nodes and the fusion centers in each cluster (represented by arrows with two peaks).

Moreover, any global coordination is not required since all clusters can transmit simultaneously.

The attentive reader, however, could conclude from Theorem 3 that in each cluster, say cluster $k$, already $\mathcal{O}\left(2\left|C_{k}\right|+1\right)$ wireless resource units are sufficient to universally compute every $f_{k} \in \mathcal{C}^{0}\left[\mathbb{E}^{\left|C_{k}\right|}\right], k=1, \ldots, K$, over the $f$-MAC (i.e., by harnessing interference). Since $\max _{1 \leq k \leq K}\left\{L_{k}\right\}=\max _{1 \leq k \leq K}\left\{2\left|C_{k}\right|+1\right\} \leq 2 N+1$ holds, seemingly further wireless resources could be saved. Because of the couplings between clusters due to common nodes, however, this would require a constant adaptation of the pre-processing on the common sensor nodes and a large amount of coordination. In order to illustrate this please remind that there exist homeomorphisms $\left(x_{k_{1}}, \ldots, x_{k_{\mid C_{k}}}\right) \mapsto\left(y_{k}(1), \ldots, y_{k}\left(2\left|C_{k}\right|+1\right)\right)$ between $\mathbb{E}^{\mid C_{k}}$ and $\Gamma_{k} \subset \mathbb{R}^{2\left|C_{k}\right|+1}$ that enable each cluster to compute every $f_{k} \in \mathcal{C}^{0}\left[\mathbb{E}^{\mid C_{k}}\right]$ because of the existence of representations

$$
f_{k}\left(x_{k_{1}}, \ldots, x_{k_{\left|C_{k}\right|}}\right)=\sum_{\ell=1}^{2\left|C_{k}\right|+1} \psi_{k \ell}\left(\sum_{n \in C_{k}} \varphi_{n \ell}^{(k)}\left(x_{n}\right)\right),
$$

$k=1, \ldots, K$. Since the compact sets $\Gamma_{k}$ will unfortunately differ in general, the pre-processing functions in (21) depend also on $k$. For nodes whose transmit signals can only be received by a single fusion center it does not matter. But nodes that can be heard by more than one fusion center (i.e., the common nodes between clusters) have to adapt their pre-processing functions in dependency of the fusion center which they want to address. As well as in the case of TDMA this in turn would require the separated activation of clusters (see Fig. 6) such that $\mathcal{O}\left(\sum_{k=1}^{K}\left(2\left|C_{k}\right|+1\right)\right)$ resource units are necessary.

\section{Robustness to Changing Topologies}

In Theorem 1, we have shown that every $f \in \mathcal{F}\left[\mathbb{E}^{N}\right]$ is computable over an $f$-MAC of order 1 . The surprising fact is that the computations are universal (see Definition 4) and thus pre-processing functions are independent of the desired function. However, the corresponding proof relies on a mentioned result by Buck in [35] that depends on the number $N$ of active nodes. As a consequence, transmitting sensors have to adapt their pre-processing functions if the network topology changes (i.e., the universality is not robust against modified $N$ ), which 
would be highly undesired in sensor networks. Hence, we want to know if this holds in general and analyze therefore in this section the robustness of universality against variations in network topology due to sensor nodes that drop out of the network or due to new sensor nodes that join the network.

\section{A. Dropped Out Nodes}

Let us first consider the case where a number of sensor nodes drop out of the network due to for instance failures or battery depletion. The question is whether the universality property is preserved when an arbitrary subset of nodes leaves the network. The following theorem gives the answer.

Theorem 4: The universality of analog computation via an $f$-MAC of order 1 is robust against dropped nodes.

Proof: The proof is deferred to Appendix B.

It should be emphasized that even if according to Theorem 4 the pre-processing functions do not depend on the desired function and the number of active nodes (i.e., remaining nodes have not to be updated if other nodes failed), the post-processing function does.

\section{B. Additional Nodes}

We now consider the opposite case where an existing sensor network for computation purposes is enlarged by adding a finite number of active nodes. More precisely, assume that we connect $J-N \in \mathbb{N}, N<J<\infty$, transmitting sensor nodes to the network to universally compute every desired function $f\left(x_{1}, \ldots, x_{N}, \ldots, x_{J}\right) \in \mathcal{F}\left[\mathbb{E}^{J}\right]$ of the measurements. Then, we want to answer the question if the universality is preserved if the existing $N$ active nodes were already able to universally compute every $f\left(x_{1}, \ldots, x_{N}\right) \in \mathcal{F}\left[\mathbb{E}^{N}\right]$.

Theorem 5: The universality of analog computation via an $f$-MAC of order 1 is robust against a fixed enlargement of the network.

Proof: The proof is deferred to Appendix C.

Observation 3: It is not necessary to update the existing transmitting nodes (i.e., the pre-processing) if the network is enlarged by adding further active transmitting nodes.

Note that the term "fixed" in Theorem 5 as well as the idea of proof refers to the fact that in the current form, the robustness of universality holds if the original network was already designed for $J>N$ nodes but only $N$ nodes are deployed to the measuring field. Then, adding up to $J-N$ nodes during network operation has no impact on the previous $N$ nodes. The more general case in which the original network was designed for at most $N$ nodes but extended to $J$ nodes afterwards is therefore still an open problem.

It should be emphasized, however, that this limits the practical significance of Theorem 5 only marginally. A robust network for computation purposes can always be designed without knowing the exact number $J-N$ in advance by choosing $J$ sufficiently large (according to the application needs) and using only $N$ out of $J$ nodes in practice.

Remark 12: Note that even if Theorems 4 and 5 refer to single-cluster networks, they remain valid for arbitrary networks of $f$-MACs of order 1 as well. The only small difference is that if common nodes drop out of the network, then all affected fusion centers have to update their post-processing functions. On the other hand, in a network of $f$-MACs of order
$2 N+1$, where continuous pre- and post-processing functions are employed, the fusion centers have to additionally adjust the correction terms (15) by appropriately adding further constants that correspond to the dropped out nodes.

\section{SOME REMARKS ON NON-IDEAL CHANNELS}

The assumption of an ideal wireless MAC model in the previous sections, while being restrictive in terms of practical applications, makes an in-depth and rigorous analysis possible. The analysis provides interesting insights into the limits of analog function computations in wireless networks; it further sheds light on potential research directions. Practical computation schemes will, however, suffer from a number of impairments, including the lack of synchronization or fading. Therefore, in this section, we demonstrate potential benefits of our results by presenting two numerical experiments carried out based on the practical analog computation scheme proposed in [5], [6], [34], [46]. This scheme is robust against both asynchronism and, under certain assumption, fading effects.

Consider (1) in the complex baseband with sequences of transmit symbols given by

$$
s_{n \ell}^{(k)}\left(x_{n}\right)=\sqrt{\frac{\theta\left(\varphi_{n}\left(x_{n}\right)\right)}{L}} d_{k n}(\ell), \quad \ell=1, \ldots, L,
$$

$n \in C_{k}$, where $d_{k n}(\ell)$ is randomly drawn from $\{-1,1\}$, for all $k, n, \ell$. The (affine) map $\theta: \mathbb{E} \rightarrow[0, P]$, which is assumed to be known to the fusion centers, ensures that each symbol fulfills some transmit power constraint $P>0$ [46]. The complex-valued frequency-flat channel coefficients are of the form

$$
h_{k n}(\ell):=\sqrt{r_{k n}^{-\alpha}} \tilde{h}_{k n}(\ell)
$$

Here, $r_{k n}$ is the spatial distance between node $n \in C_{k}$ and the $k$ th fusion center, $\alpha=2$ is the path loss exponent and $\tilde{h}_{k n}(\ell)$ is on independent identically distributed Rayleigh fading with unit variance, for all $k, n, \ell$. Finally, we model the receiver noise in (1) as proper complex Gaussian with unit power spectral density.

Using the wireless MAC $L$ times as in (22) has the advantage that a precise symbol and phase synchronization for a constructive superposition of transmit signals in the sense of (1) is not necessary. Moreover, obtaining a function value at a fusion center reduces to a simple estimation of the receive energy [46]. Note that according to the system model (see Remark 1), the distances $r_{k n}$ for $n \notin C_{k}$ are large enough to assume that the corresponding contribution to the receive energy is negligible.

We compare the described practical computation scheme with a simplified standard TDMA approach in which the nodes transmit symbols of the form $s_{n \ell}^{(k)}\left(x_{n}\right)=\sqrt{\theta\left(\varphi_{n}\left(x_{n}\right)\right)} d_{k n}(\ell)$ in a time-sharing fashion, with $d_{k n}(\ell)$ as defined above. To make the comparison fair, the transmit power in (22) is normalized by the number of channel uses $L$ so that both schemes utilize the same transmit energy per function computation.

Due to the stochastic nature of the underlying non-ideal MAC, we have to evaluate the schemes using probabilistic tools and metrics. In particular, the computation performance 


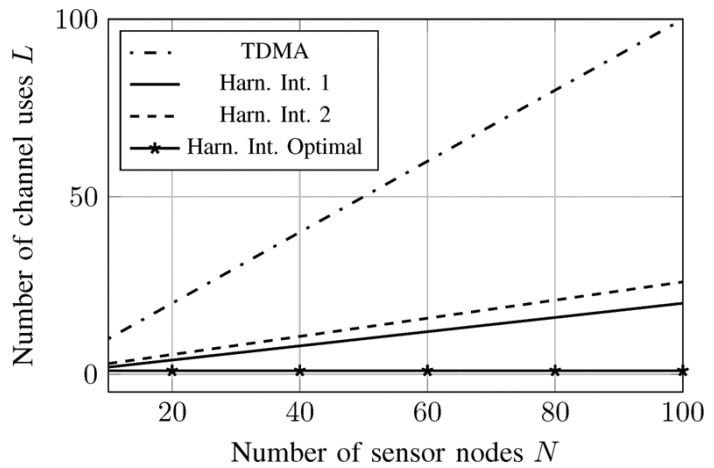

Fig. 7. Harnessing interference for analog function computations without any CSI vs. TDMA with and without CSI. The depicted numbers of channel uses for harnessing interference are the smallest $L$ for which the mean square error is smaller than or equal to that of TDMA with perfect CSI at nodes (dashed line) or without CSI at nodes (solid line).

at the $k$ th fusion center, $k=1, \ldots, K$, is expressed in terms of the mean square error defined to be

$\operatorname{MSE}_{k}:=\mathrm{E}\left\{\left(\hat{f}\left(x_{k_{1}}, \ldots, x_{k_{\left|C_{k}\right|}}\right)-f\left(x_{k_{1}}, \ldots, x_{k_{\left|C_{k}\right|}}\right)\right)^{2}\right\}$.

Note that in the case of (22), the MSE is a function of $L$.

Example 4: Consider a single cluster with $N=$ $10,15, \ldots, 100$ nodes that are located at equal unit distances to the fusion center. Let the sensor readings be independent and uniformly drawn from $\mathbb{E}$, the desired function be chosen to be the arithmetic mean (see Example (1)(i)) and assume that transmissions are performed in the very low power regime as it is typical for wireless sensor networks. The corresponding experimental data as a result of $10^{5}$ Monte Carlo runs (i.e., computations) is depicted in Fig. 7. The plots for the practical harnessing interference approach result from considering for each given $N$ the smallest $L$ for which the mean square error is smaller or equal to that of TDMA with Channel State Information (CSI) at nodes or to that of TDMA without CSI.

Even though the practical scheme from [46] does not attains the optimum in Fig. 7, it demonstrates the advantage of approaches based on harnessing interference for computations over approaches that avoid the interference through orthogonal transmissions. The TDMA approach can compute a function value after $N$ channel uses, whereas the computation scheme that harnesses interference allows function value computations approximately after $N / 5$ channel uses at a comparable mean square error performance without any CSI. If for TDMA each node has perfect CSI prior to transmissions, then approximately $N / 4$ channel uses are sufficient to achieve the same mean square error performance by harnessing interference.

Example 5: Consider a network of $N=300$ nodes that are organized into $K=3$ clusters with 100 nodes per cluster (i.e., $\left.\left|C_{1}\right|=\left|C_{2}\right|=\left|C_{3}\right|=100\right)$. Furthermore, let $\left|C_{1} \cap C_{2}\right|=15$, $\left|C_{2} \cap C_{3}\right|=30$, and let the distances $r_{n k}$ between nodes and fusion centers be uniformly drawn from [1 m $10 \mathrm{~m}]$; all other simulation parameters are as in Example 4. The corresponding experimental data is depicted in Fig. 8.

The plots indicate that when using a standard TDMA for function computations in the network of Example 5, there are

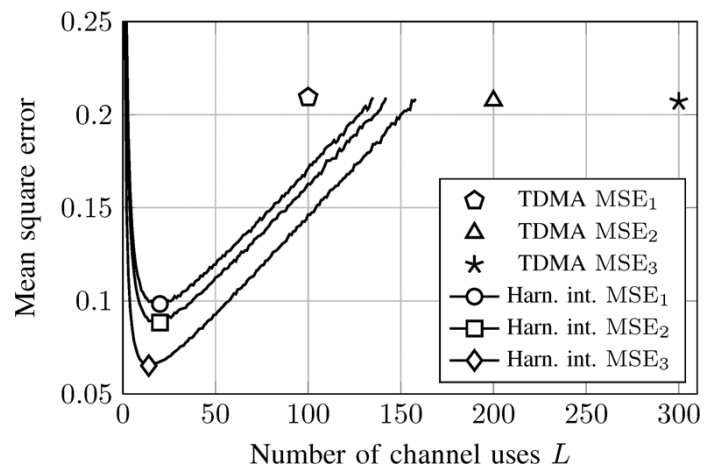

Fig. 8. Harnessing interference for analog function computations with statistical CSI vs. TDMA in a network of $N=300$ nodes, $K=3$ clusters and 100 nodes per cluster. Clusters 1 and 2 share 15 nodes, whereas clusters 2 and 3 share 30 nodes.

at least 300 channel uses necessary until each fusion center can complete the current computation round. On the other hand, harnessing interference with some statistical a priori channel knowledge at the fusion centers achieves in each cluster a significantly smaller mean square error performance than TDMA with the number of channel uses reduced by a factor of $\approx 17$. In [34] it was shown that providing statistical CSI to the fusion centers is much less involved than providing instantaneous CSI to individual sensor nodes.

Remark 13: The reason for the monotonically increasing mean square error (after reaching a minimum) in Fig. 8 is the normalization of the transmit power in (22) by $L$, which results in lower signal-to-noise ratios when $L$ increases.

Remark 14: It is important to emphasize that the analog transmission scheme used to obtain the above numerical examples is only one possible approach to the problem of harnessing interference for function computation in wireless networks.

\section{CONCLUSION}

In this paper, we studied the problem of analog-computing functions at fusion centers in clustered wireless sensor networks, where nodes transmit simultaneously to harness the interference property of the wireless channel. By applying appropriate pre-processing functions on sensor readings and post-processing functions on the superimposed signals received by the fusion centers, in addition to linear functions even nonlinear functions are computable over the channel.

If no restrictions on pre- and post-processing functions are imposed, we have shown that in fact every function can be computed on each fusion center, where the number of required wireless resource units is of the order $\mathcal{O}(1)$, whereas a standard TDMA requires $\mathcal{O}(K M)$. The latter scales with the number $K$ of clusters and the number $M$ of nodes belonging to the largest cluster such that huge performance gains are possible if computations are performed over the channel.

Although implementing continuous pre- and post-processing functions in practice is generally less complex, a corresponding restriction generates the need for an additional post-processing step at fusion centers to ensure the computability of at least every continuous function of sensor readings. However, requiring pre- and post-processing functions to be continuous generally needs additional wireless resources. In particular, 
we have shown that the number of required resource units to simultaneously compute any continuous functions at distinct fusion centers is then of the order $\mathcal{O}(2 N+1)$. Since this is proportional to the number $N$ of nodes but not to the number of clusters, the proposed computation scheme still offers significant performance gains in comparison to a standard TDMA.

A remarkable property of analog computations over the channel is that they can always be universally performed. Universally means that pre-processing functions are independent of the functions to be computed at fusion centers such that they do not need to be updated if desired functions change. This implies that the feedback overhead between nodes and fusion centers can be reduced since corresponding coordination is not needed. Therefore, the architecture of sensor nodes for computation purposes is universal and of reduced complexity, which make them cheap and easy to handle. In this regard, we have shown that the universality property is even preserved if the network topology varies because of nodes that leave or enter the network.

The work in this paper demonstrates that analog systems are well suited to efficiently solve arbitrary computation problems in sensor networks. Indeed, it was recently even shown in [47] that with ordinary sampling, purely analog linear systems are not always stably representable in discrete time domain. Moreover, sampling is usually followed by quantization, which generates additional instabilities that are not always controllable by oversampling [48], [49]. Thus, digital signal processing has some fundamental limits and analog systems are gaining more attention in the sensor network community.

A promising application of the results in this paper can be the efficient implementation of wireless neural networks since for example in [50], [51] it is shown that Kolmogorov's solution to Hilbert's 13th problem is relevant for neurocomputing.

Remark 15: Finally, we point out that we considered sensor readings which are drawn from the unit interval $\mathbb{E}$. This, however, is no loss in generality since all statements remain valid for arbitrary compact metric spaces. For example, Theorem 3 can also be proven by using a result from [52] that extends Kolmogorov's refutation of Hilbert's 13th problem to arbitrary compact metric spaces of appropriate covering dimension.

\section{APPENDIX A}

\section{A. Proof of Lemma 1}

The proof is a generalization of an idea from [53].

" $\Leftarrow$ ": Let $\boldsymbol{x}^{(1)}, \boldsymbol{x}^{(2)} \in \mathbb{E}^{N}$, with $\boldsymbol{x}^{(1)} \neq \boldsymbol{x}^{(2)}$. Since $g$ is bijective, it follows that $g\left(\boldsymbol{x}^{(1)}\right) \neq g\left(\boldsymbol{x}^{(2)}\right)$ and from the fact that $\mathbb{E}^{N}$ has the cardinality of the continuum, $\Upsilon$ has the cardinality of the continuum as well.

Now, let $g(\boldsymbol{x})=: x \in \Upsilon$ and $g^{*}$ be a function such that $g^{*} \circ g=\mathrm{id}_{\mathbb{E}^{N}}$, that is

$$
g^{*}: \Upsilon \rightarrow \mathbb{E}^{N}, g^{*}(x)=\left(\begin{array}{c}
g_{1}^{*}(x)=x_{1} \\
\vdots \\
g_{N}^{*}(x)=x_{N}
\end{array}\right)
$$

Then, we conclude with $\psi:=f \circ g^{*}$

$$
\begin{aligned}
f\left(x_{1}, \ldots, x_{N}\right) & =f\left(g_{1}^{*}(x), \ldots, g_{N}^{*}(x)\right)=\left(f \circ g^{*}\right)(x) \\
& =\psi(x)=\psi\left(g\left(x_{1}, \ldots, x_{N}\right)\right) \\
& =\psi\left(\sum_{n=1}^{N} \varphi_{n}\left(x_{n}\right)\right) .
\end{aligned}
$$

" $\Rightarrow$ ": If $g$ is not bijective, there exist at least two points $\boldsymbol{x}^{(1)}, \boldsymbol{x}^{(2)} \in \mathbb{E}^{N}$, with $\boldsymbol{x}^{(1)} \neq \boldsymbol{x}^{(2)}$ but $g\left(\boldsymbol{x}^{(1)}\right)=g\left(\boldsymbol{x}^{(2)}\right)$, as well as an $f \in \mathcal{F}\left[\mathbb{E}^{N}\right]$ with $f\left(\boldsymbol{x}^{(1)}\right) \neq f\left(\boldsymbol{x}^{(2)}\right)$. This, however, leads to a contradiction because of $f\left(\boldsymbol{x}^{(1)}\right)=\psi\left(g\left(\boldsymbol{x}^{(1)}\right)\right)=$ $\psi\left(g\left(\boldsymbol{x}^{(2)}\right)\right)=f\left(\boldsymbol{x}^{(2)}\right)$, from which follows that $\left\{\varphi_{n}\right\}_{1 \leq n \leq N}$ are not universal pre-processing functions in the sense of Definition 4.

\section{B. Proof of Theorem 4}

Let $N \geq 2$ and $\varphi_{1}, \ldots, \varphi_{N} \in \mathcal{F}[\mathbb{E}]$ be fixed universal pre-processing functions to compute every $f\left(x_{1}, \ldots, x_{N}\right) \in$ $\mathcal{F}\left[\mathbb{E}^{N}\right]$. Furthermore, let $\mathcal{I} \neq \varnothing$ be any subset of $\mathcal{I}_{N}:=\{1, \ldots, N\}$. Then, we have to prove that $\left\{\varphi_{k}\right\}_{k \in \mathcal{I}}$ are also universal pre-processing functions to compute every $f\left(x_{1}, \ldots, x_{k}\right) \in \mathcal{F}\left[\mathbb{E}^{k}\right], k \in \mathcal{I}$. Since the problem is permutation invariant, the numbering of nodes does not matter. Hence, we assume $\mathcal{I}=\mathcal{I}_{\ell}=\{1, \ldots, \ell\}$ with $\ell<N$, since otherwise there is nothing to prove.

If we proceed inductively, we have to show that $\varphi_{1}, \ldots, \varphi_{N-1}$ are universal pre-processing functions for nodes $\{1, \ldots, N-1\}$ to compute every $f\left(x_{1}, \ldots, x_{N-1}\right) \in \mathcal{F}\left[\mathbb{E}^{N-1}\right]$. If this is successful, we arrive in $N-\ell$ steps at $\mathcal{I}_{\ell} \subset \mathcal{I}_{N}$.

We prove the induction hypothesis by contradiction. Assume $\varphi_{1}, \ldots, \varphi_{N-1}$ are not universal pre-processing functions. Then, due to Lemma $1, \sum_{n=1}^{N-1} \varphi_{n}\left(x_{n}\right)$ is not bijective and hence there exist at least two points $\boldsymbol{x}^{(1)}:=\left(x_{1}^{(1)}, \ldots, x_{N-1}^{(1)}\right) \in \mathbb{E}^{N-1}$ and $\boldsymbol{x}^{(2)}:=\left(x_{1}^{(2)}, \ldots, x_{N-1}^{(2)}\right) \in \mathbb{E}^{N-1}, \boldsymbol{x}^{(1)} \neq \boldsymbol{x}^{(2)}$, such that

$$
\sum_{n=1}^{N-1} \varphi_{n}\left(x_{n}^{(1)}\right)=\sum_{n=1}^{N-1} \varphi_{n}\left(x_{n}^{(2)}\right) .
$$

Now, we choose an arbitrary $\hat{x}_{N} \in(0,1)$ and consider the points $\hat{\boldsymbol{x}}^{(1)}:=\left(\begin{array}{c}\boldsymbol{x}^{(1)} \\ \hat{x}_{N}\end{array}\right)$ and $\hat{\boldsymbol{x}}^{(2)}:=\left(\begin{array}{c}\boldsymbol{x}^{(2)} \\ \hat{x}_{N}\end{array}\right)$. Of course $\hat{\boldsymbol{x}}^{(1)} \neq$ $\hat{\boldsymbol{x}}^{(2)}$ and therefore

$$
\begin{aligned}
\sum_{n=1}^{N} \varphi_{n}\left(\hat{x}_{n}^{(1)}\right) & =\sum_{n=1}^{N-1} \varphi_{n}\left(x_{n}^{(1)}\right)+\varphi_{N}\left(\hat{x}_{N}\right) \\
& =\sum_{n=1}^{N-1} \varphi_{n}\left(x_{n}^{(2)}\right)+\varphi_{N}\left(\hat{x}_{N}\right)=\sum_{n=1}^{N} \varphi_{n}\left(\hat{x}_{n}^{(2)}\right)
\end{aligned}
$$

contradicts the universality of $\varphi_{1}, \ldots, \varphi_{N}$ when $N$ nodes are active, which proves the preservation of universality for $\mathcal{I}_{N} \rightarrow$ $\mathcal{I}_{N-1}$. Proceeding essentially along the same lines shows that the property is preserved for $\mathcal{I}_{N-1} \rightarrow \mathcal{I}_{N-2} \rightarrow \cdots \rightarrow \mathcal{I}_{1}$.

\section{Proof of Theorem 5}

The proof follows immediately from the proof of Theorem 4 by setting $N=J$ and by considering all subsets $\mathcal{I}_{\ell}=\{1, \ldots, \ell\}$ of $\mathcal{I}_{J}=\{1, \ldots, J\}$, with $N \leq \ell<J$. 
Starting with the assumption that $\left\{\varphi_{k}\right\}_{1<k<J}$ are fixed universal pre-processing functions to compute every $f \in \mathcal{F}\left[\mathbb{E}^{J}\right]$, the induction arrives in $J-N$ steps at $\ell=N$ such that $\left\{\varphi_{k}\right\}_{1 \leq k \leq \ell}$ are universal pre-processing functions to compute every $f \in \mathcal{F}\left[\mathbb{E}^{\ell}\right]$, for all $N \leq \ell \leq J$.

\section{ACKNOWLEDGMENT}

The authors would like to thank the anonymous reviewers for their helpful comments and suggestions.

\section{REFERENCES}

[1] F. Zhao and L. Guibas, Wireless Sensor Networks: An Information Processing Approach. San Francisco, CA, USA: Elsevier/Morgan Kaufmann, 2004.

[2] R. F. Wyrembelski and H. Boche, "Physical layer integration of private, common, and confidential messages in bidirectional relay networks," IEEE Trans. Wireless Commun., vol. 11, no. 9, pp. 3170-3179, Sep. 2012.

[3] A. Giridhar and P. R. Kumar, "Toward a theory of in-network computation in wireless sensor networks," IEEE Commun. Mag., vol. 44, no. 4, pp. 98-107, Apr. 2006.

[4] M. Gastpar and M. Vetterli, "Source-channel communication in sensor networks," in Proc. 2nd Int. Workshop Inf. Process. Sensor Netw. (IPSN), Palo Alto, CA, USA, Apr. 2003, pp. 162-177.

[5] M. Goldenbaum, S. Stańczak, and M. Kaliszan, "On function computation via wireless sensor multiple-access channels," in Proc. IEEE Wireless Commun. Netw. Conf. (WCNC), Budapest, Hungary, Apr. 2009.

[6] M. Goldenbaum and S. Stańczak, "Computing the geometric mean over multiple-access channels: Error analysis and comparisons," in Proc. 44th Asilomar Conf. Signals, Syst., Comput., Pacific Grove, CA, USA, Nov. 2010, pp. 2172-2178.

[7] G. Mergen and L. Tong, "Type based estimation over multiaccess channels," IEEE Trans. Signal Process., vol. 54, no. 2, pp. 613-626, Feb. 2006.

[8] W. U. Bajwa, J. D. Haupt, A. M. Sayeed, and R. D. Nowak, "Joint source-channel communication for distributed estimation in sensor networks," IEEE Trans. Inf. Theory, vol. 53, no. 10, pp. 3629-3653, Oct. 2007.

[9] J.-J. Xiao, S. Cui, Z.-Q. Luo, and A. J. Goldsmith, "Linear coherent decentralized estimation," IEEE Trans. Signal Process., vol. 56, no. 2, pp. 757-770, Feb. 2008.

[10] M. K. Banavar, C. Tepedelenlioğlu, and A. Spanias, "Estimation over fading channels with limited feedback using distributed sensing," IEEE Trans. Signal Process., vol. 58, no. 1, pp. 414-425, Jan. 2010.

[11] S. Stańczak, M. Wiczanowski, and H. Boche, "Distributed power control for optimizing a weighted sum of link-layer QoS levels," in Proc. IEEE Global Telecommun. Conf. (GLOBECOM), St. Louis, MO, USA, Nov. 2005, pp. 3539-3543.

[12] T. M. Duman and M. Salehi, "Decentralized detection over multipleaccess channels," IEEE Trans. Aerosp. Electron. Syst., vol. 34, no. 2, pp. 469-476, Apr. 1998.

[13] W. Li and H. Dai, "Distributed detection in wireless sensor networks using a multiple access channel," IEEE Trans. Signal Process., vol. 55, no. 3, pp. 822-833, Mar. 2007.

[14] G. Mergen, V. Naware, and L. Tong, "Asymptotic detection performance of type-based multiple access over multiaccess fading channels," IEEE Trans. Signal Process., vol. 55, no. 3, pp. 1081-1092, Mar. 2007.

[15] K. Liu and A. M. Sayeed, "Type-based decentralized detection in wireless sensor networks," IEEE Trans. Signal Process., vol. 55, no. 5, pp. 1899-1910, May 2007.

[16] M. K. Banavar, A. D. Smith, C. Tepedelenlioğlu, and A. Spanias, "On the effectiveness of multiple antennas in distributed detection over fading MACs," IEEE Trans. Wireless Commun., vol. 11, no. 5, pp. 1744-1752, May 2012.

[17] F. Li, J. S. Evans, and S. Dey, "Design of distributed detection schemes for multiaccess channels," IEEE Trans. Aerosp. Electron. Syst., vol. 48, no. 2, pp. 1552-1569, Apr. 2012.

[18] P. Jakimovski, F. Becker, S. Sigg, H. R. Schmidtke, and M. Beigl, "Collective communication for dense sensing environments," in Proc. 7th Int. Conf. Intell. Environments (IE), Nottingham, U.K., July 2011, pp. 157-164.
[19] S. Sigg, P. Jakimovski, and M. Beigl, "Calculation of functions on the RF-channel for IoT," in Proc. 3rd Int. Conf. Internet of Things (IOT), Wuxi, China, Oct. 2012, pp. 107-113.

[20] S. Boyd, A. Ghosh, B. Prabhakar, and D. Shah, "Randomized gossip algorithms," IEEE Trans. Inf. Theory, vol. 52, no. 6, pp. 2508-2530, June 2006

[21] R. Olfati-Saber, J. A. Fax, and R. M. Murray, "Consensus and cooperation in networked multi-agent systems," Proc. IEEE, vol. 95, no. 1, pp. 215-233, Jan. 2007.

[22] D. Mosk-Aoyama and D. Shah, "Fast distributed algorithms for computing separable functions," IEEE Trans. Inf. Theory, vol. 54, no. 7, pp. 2997-3007, July 2008.

[23] A. G. Dimakis, S. Kar, J. M. F. Moura, M. G. Rabbat, and A. Scaglione, "Gossip algorithms for distributed signal processing," Proc. IEEE, vol. 98, no. 11, pp. 1847-1864, Nov. 2010.

[24] B. Nazer, A. G. Dimakis, and M. Gastpar, "Local interference can accelerate gossip algorithms," IEEE J. Sel. Topics Signal Process., vol. 5, no. 4, pp. 876-887, Aug. 2011.

[25] A. Orlitsky and J. R. Roche, "Coding for computing," IEEE Trans. Inf. Theory, vol. 47, no. 3, pp. 903-917, Mar. 2001.

[26] V. Doshi, D. Shah, M. Médard, and M. Effros, "Functional compression through graph coloring," IEEE Trans. Inf. Theory, vol. 56, no. 8, pp. 3901-3917, Aug. 2010.

[27] B. Nazer and M. Gastpar, "Computation over multiple-access channels," IEEE Trans. Inf. Theory, vol. 53, no. 10, pp. 3498-3516, Oct. 2007.

[28] M. Goldenbaum, H. Boche, and S. Stańczak, "Reliable computation of nomographic functions over Gaussian multiple-access channels," in Proc. IEEE Int. Conf. Acoust., Speech, Signal Process. (ICASSP), Vancouver, Canada, May 2013, pp. 4814-4818.

[29] L. Keller, N. Karamchandani, and C. Fragouli, "Function computation over linear channels," in Proc. IEEE Int. Symp. Netw. Coding (NetCod), Toronto, ON, Canada, June 2010, pp. 1-6.

[30] A. N. Kolmogorov, "On the representation of continuous functions of several variables by superposition of continuous functions of one variable and addition," Dokl. Akad. Nauk SSSR, no. 114, pp. 953-956, 1957, engl. translation.

[31] D. Hilbert, "Mathematical problems," Bull. Amer. Math. Soc., vol. 8, no. 10, pp. 437-479, 1902.

[32] S. Verdú, Multiuser Detection. Cambridge, U.K.: Cambridge Univ. Press, 1998.

[33] D. Tse and P. Viswanath, Fundamentals of Wireless Communications. Cambridge, U.K.: Cambridge Univ. Press, 2005.

[34] M. Goldenbaum and S. Stańczak, "Computing functions via SIMO multiple-access channels: How much channel knowledge is needed?," in Proc. IEEE Int. Conf. Acoust., Speech, Signal Process. (ICASSP), Dallas, TX, USA, Mar. 2010, pp. 3394-3397.

[35] R. C. Buck, "Approximate complexity and functional representation," J. Math. Anal. Appl., vol. 70, pp. 280-298, 1979.

[36] L. I. Epstein, Nomography. New York, NY, USA: Interscience Publishers, 1958.

[37] D. A. Sprecher, "A representation theorem for continuous functions of several variables," Amer. Math. Soc., vol. 16, no. 2, pp. 200-203, 1965.

[38] J. V. Neumann, "Ein System algebraisch unabhängiger Zahlen," Math. Ann., vol. 99, pp. 134-141, 1928.

[39] R. C. Buck, "Nomographic functions are nowhere dense," in Proc. Amer. Math. Soc., June 1982, vol. 85, no. 2, pp. 195-199.

[40] V. I. Arnol'd, "On the representation of functions of two variables in the form $\chi[\varphi(x)+\psi(y)]$," Uspekhi Math. Nauk, vol. 12, no. 2, pp. 119-121, 1957, engl. translation.

[41] A. G. Vitushkin, "On Hilbert's thirteenth problem and related questions," Russian Math. Surveys, vol. 59, no. 1, pp. 11-25, 2004.

[42] J. Braun and M. Griebel, "On a constructive proof of Kolmogorov's superposition theorem," Constr. Approx., vol. 30, no. 3, pp. 653-675, Dec. 2009.

[43] W. Hurewicz and H. Wallman, Dimension Theory. Princeton, NJ, USA: Princeton Univ. Press, 1948.

[44] Y. Sternfeld, "Dimension, superposition of functions and separation of points, in compact metric spaces," Israel J. Math., vol. 50, pp. 13-52, 1985.

[45] A. G. Vitushkin and G. M. Khenkin, "Linear superpositions of functions," Russian Math. Surveys, vol. 22, no. 1, pp. 77-125, 1967.

[46] M. Goldenbaum and S. Stańczak, "Robust analog function computation via wireless multiple-access channels," IEEE Trans. Commun. 2012 [Online]. Available: http://arxiv.org/abs/1210.2967, [Online]. Available: 
[47] H. Boche and U. J. Mönich, "Sampling of deterministic signals and systems," IEEE Trans. Signal Process., vol. 59, no. 5, pp. 2101-2111, May 2011.

[48] H. Boche and U. J. Mönich, "Behavior of the quantization operator for bandlimited, nonoversampled signals," IEEE Trans. Inf. Theory, vol. 56, no. 5, pp. 2433-2440, May 2010.

[49] H. Boche and U. J. Mönich, "Unboundedness of thresholding and quantization for bandlimited signals," Signal Process., vol. 92, no. 12, pp. 2821-2829, Dec. 2012.

[50] R. Hecht-Nielsen, "Kolmogorov's mapping neural network existence theorem," in Proc. IEEE 1st Int. Conf. Neural Netw., San Diego, CA, USA, June 1987, vol. III, pp. 11-14.

[51] V. Kurková, "Kolmogorov's theorem and multilayer neural networks," Neural Netw., vol. 5, no. 3, pp. 501-506, 1992.

[52] P. A. Ostrand, "Dimension of metric spaces and Hilbert's problem 13," Bull. Amer. Math. Soc., vol. 71, no. 4, pp. 619-622, July 1965.

[53] L. Bieberbach, "Operationsbereiche von Funktionen," in Proc. Int. Congr. Mathematicians (ICM), Zürich, Switzerland, Sept. 1932, vol. I, pp. 162-172.

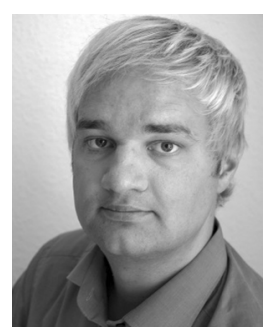

Mario Goldenbaum (S'09) received the Dipl.-Ing. (FH) and Dipl.-Ing. degree in electrical engineering and information technology from the Bremen University of Applied Sciences and the University of Bremen, Germany, in 2005 and 2007, respectively. In 2008, he joined the Fraunhofer Institute for Telecommunications, Heinrich Hertz Institute (HHI), Berlin, Germany, as a research associate. Since 2010 , he works as a research and teaching assistant at the Fachgebiet für Informationstheorie und theoretische Informationstechnik, Technische Universität Berlin, Germany, and is pursuing his Ph.D. degree at the Lehrstuhl für Theoretische Informationstechnik, Technische Universität München, Munich, Germany.

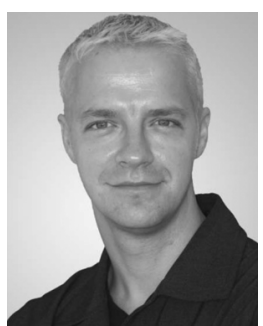

Holger Boche (M'04-SM'07-F'11) received the Dipl.-Ing. and Dr.-Ing. degrees in electrical engineering from the Technische Universität Dresden, Dresden, Germany, in 1990 and 1994, respectively. He graduated in mathematics from the Technische Universität Dresden in 1992. From 1994 to 1997, he did postgraduate studies in mathematics at the Friedrich-Schiller Universität Jena, Jena, Germany. He received his Dr. rer. nat. degree in pure mathematics from the Technische Universität Berlin, Berlin, Germany, in 1998. In 1997, he joined the Heinrich-Hertz-Institut (HHI) für Nachrichtentechnik Berlin, Berlin, Germany. Starting in 2002, he was a Full Professor for mobile communication networks with the Institute for Communications Systems, Technische Universität Berlin. In 2003, he became Director of the Fraunhofer German-Sino Lab for Mobile Communications, Berlin, Germany, and in 2004 he became the Director of the Fraunhofer Institute for Telecommunications (HHI), Berlin, Germany. Since October 2010 he has been with the Institute of Theoretical Information Technology and Full Professor at the Technische Universität München, Munich, Germany. He was a Visiting Professor with the ETH Zurich, Zurich, Switzerland, during the 2004 and 2006 Winter terms, and with KTH Stockholm, Stockholm, Sweden, during the 2005 Summer term. Prof. Boche is a Member of IEEE Signal Processing Society SPCOM and SPTM Technical Committee. He was elected a Member of the German Academy of Sciences (Leopoldina) in 2008 and of the Berlin Brandenburg Academy of Sciences and Humanities in 2009. He received the Research Award "Technische Kommunikation" from the Alcatel SEL Foundation in October 2003, the "Innovation Award" from the Vodafone Foundation in June 2006, and the Gottfried Wilhelm Leibniz Prize from the Deutsche Forschungsgemeinschaft (German Research Foundation) in 2008. He was corecipient of the 2006 IEEE Signal Processing Society Best Paper Award and recipient of the 2007 IEEE Signal Processing Society Best Paper Award.

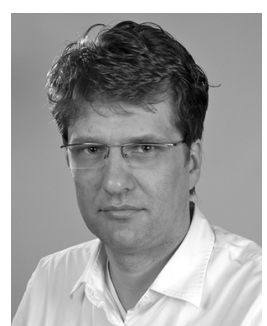

Sławomir Stańczak (M’04-SM’11) studied control systems engineering at the Wroclaw University of Technology, Poland, and at the Technische Universität Berlin (TU Berlin), Germany. He received his Dipl.-Ing. degree and Dr.-Ing. degree with distinction (summa cum laude) in electrical engineering from the TU Berlin in 1998 and 2003, respectively. Since 2006 he also holds a habilitation degree (venia legendi) and is an associate professor (Privatdozent) at the TU Berlin. Dr. Stanczak has been involved in research and development activities in wireless communications since 1997. Since 2003 he leads a research group at the Fraunhofer Heinrich Hertz Institute and since 2010 Dr. Stanczak has been the acting director of the Fachgebiet für Informationstheorie und theoretische Informationstechnik at the TU Berlin. Dr. Stanczak is a co-author of two books and more than 130 peer-reviewed journal articles and conference papers in the area of information theory, wireless communications and networking. In 2004 and 2007, he was a visiting professor at the RWTH Aachen, Germany, and, in 2008, a visiting scientist at the Stanford University, CA, USA. He is a recipient of research fellowships from the German research foundation. He was a co-chair of the 2009 International ITG Workshop on Smart Antennas (WSA 2009) and the general chair of the 2010 Workshop on Resource Allocation in Wireless Networks (RAWNET 2010). Dr. Stanczak was a co-chair of the 14th International Workshop on Signal Processing Advances in Wireless Communications (SPAWC 2013). In April 2008, he was one of three keynote speakers at the WiOpt 2008. Between 2009 and 2011, Dr. Stanczak was Associate Editor for European Transactions for Telecommunications (information theory). Since 2012, he is Associate Editor for IEEE Transactions on Signal Processing. Dr. Stanczak is a co-author of the book "Fundamentals of Resource Allocation in Wireless Networks", Springer-Verlag. 